JOURNAL OF THE AMERICAN MATHEMATICAL SOCIETY

Volume 13, Number 1, Pages 101-124

S 0894-0347(99)00318-5

Article electronically published on September 29, 1999

\title{
PRINCIPE LOCAL-GLOBAL POUR LES ZÉRO-CYCLES SUR LES SURFACES RÉGLÉES
}

\author{
JEAN-LOUIS COLLIOT-THÉLÈNE
}

Avec un appendice par E. Frossard et V. Suresh

\section{INTRODUCTION}

Soient $k$ un corps et $X$ une $k$-variété projective, lisse et géométriquement intègre. Un zéro-cycle sur $X$ est, rappelons-le, une combinaison linéaire à coefficients entiers de points fermés de $X$. On note $k(P)$ le corps résiduel en un point fermé $P \in X$. Le degré (sur $k$ ) d'un zéro-cycle $\sum n_{P} P$ est l'entier $\sum_{P} n_{P}[k(P): k] \in \mathbf{Z}$. Le groupe de Chow $\mathrm{CH}_{0}(X)$ des zéro-cycles sur $X$ est le quotient du groupe $Z_{0}(X)$ des zéro-cycles par la relation d'équivalence rationnelle. L'application degré sur les zéro-cycles passe au quotient par l'équivalence rationnelle. On note $A_{0}(X) \subset \mathrm{CH}_{0}(X)$ le sousgroupe des classes de zéro-cycles de degré zéro et $\operatorname{Br}(X)=H_{\text {ét }}^{2}\left(X, \mathbb{G}_{m}\right)$ le groupe de Brauer de $X$. On note $\alpha(P) \in \operatorname{Br}(k(P))$ l'évaluation en $P$ d'un élément $\alpha \in \operatorname{Br}(X)$ et $(\alpha, P)=\operatorname{Cores}_{k(P) / k}(\alpha(P)) \in \operatorname{Br}(k)$. En envoyant le couple $\left(\alpha, \sum_{P} n_{P} P\right)$ sur $\sum_{P} n_{P}(\alpha, P) \in \operatorname{Br}(k)$, on définit un accouplement $\operatorname{Br}(X) \times Z_{0}(X) \rightarrow \operatorname{Br}(k)$ qui passe au quotient par l'équivalence rationnelle et définit un accouplement biadditif $\operatorname{Br}(X) \times \mathrm{CH}_{0}(X) \rightarrow \mathrm{Br}(k)$.

Supposons que $k$ est un corps de nombres. Soit $\Omega$ l'ensemble de ses places. Soit $k_{v}$ le complété de $k$ en $v \in \Omega$. Notons $X_{v}=X \times_{k} k_{v}$. Le groupe de $\operatorname{Brauer} \operatorname{Br}\left(k_{v}\right)$ s'injecte dans $\mathbf{Q} / \mathbf{Z}$ par l'invariant inv $v$ de la théorie du corps de classes local. Ceci définit pour chaque place $v$ un accouplement biadditif $\operatorname{Br}\left(X_{v}\right) \times \mathrm{CH}_{0}\left(X_{v}\right) \rightarrow \mathbf{Q} / \mathbf{Z}$. Un élément de $\operatorname{Br}(X)$ s'annule sur $\mathrm{CH}_{0}\left(X_{v}\right)$ pour presque toute place $v$. On a donc un accouplement biadditif

$$
\operatorname{Br}(X) \times \prod_{v \in \Omega} \mathrm{CH}_{0}\left(X_{v}\right) \rightarrow \mathbf{Q} / \mathbf{Z}
$$

Received by the editors May 29, 1998 and, in revised form, June 17, 1999.

2000 Mathematics Subject Classification. Primary 11G35, 14J26, 14C15; Secondary 14J20, $14 \mathrm{G} 25$.

La première partie de l'article (groupes de Chow relatifs, Théorèmes 1.3 et 1.4) a été conçue en janvier 1996, lors d'un séjour à l'Institut Tata (TIFR, Mumbai, Inde), Institut que j'ai plaisir à remercier pour son hospitalité. Je remercie aussi le Centre Franco-Indien pour la Promotion de la Recherche Avancée (CEFIPRA/IFCPAR) pour son soutien en diverses occasions. Le Théorème 1.5 a été trouvé à l'occasion de la conférence L'arithmétique et la géométrie des cycles algébriques, qui s'est tenue à Banff (Alberta, Canada), du 7 au 19 Juin 1998. Une version préliminaire fut mise au point à l'Institut Isaac Newton (Cambridge, G.-B.).

Je remercie R. Sujatha pour de nombreuses discussions à l'origine de ce travail, et dont on trouvera une trace au $\S 9$. Je remercie aussi E. Frossard, V. Suresh et R. Parimala pour diverses remarques. 
envoyant le couple $\left(\alpha,\left\{z_{v}\right\}_{v \in \Omega}\right)$ sur $\sum_{v \in \Omega}\left(\alpha, z_{v}\right)$. La loi de réciprocité du corps de classes global assure que l'image diagonale de $\mathrm{CH}_{0}(X)$ dans $\prod_{v \in \Omega} \mathrm{CH}_{0}\left(X_{v}\right)$ est dans le noyau de cet accouplement.

On a les conjectures suivantes ([CT95]):

Conjecture 1.1. S'il existe une famille $\left\{z_{v}\right\}_{v \in \Omega}$ de zéro-cycles de degré un qui est orthogonale à $\operatorname{Br}(X)$ par l'accouplement (1.1), alors il existe un zéro-cycle de degré un sur $X$.

Conjecture 1.2. Le complexe

$$
\varliminf_{n} A_{0}(X) / n \rightarrow \prod_{v \in \Omega} \varliminf_{n} A_{0}\left(X_{v}\right) / n \rightarrow \operatorname{Hom}(\operatorname{Br}(X), \mathbf{Q} / \mathbf{Z})
$$

est exact.

Lorsque $X$ est une courbe dont la jacobienne a un groupe de Tate-Shafarevich fini, ces conjectures sont connues. Elles sont des réinterprétations de résultats classiques ("suite duale" de Cassels-Tate). On consultera Cassels [Ca64], Manin [Ma70], [Milne86], I.6.14(b) p.102, Saito [Sai89], et [CT99].

Pour les surfaces rationnelles (surfaces birationnelles au plan projectif après extension finie du corps de base), ces conjectures avaient été proposées par Sansuc et l'auteur dans [CT/S81]. Les conjectures originales de [CT/S81] furent établies par Salberger [Sal88] pour les surfaces fibrées en coniques au-dessus de la droite projective $\mathbf{P}_{k}^{1}$ (ce sont des surfaces rationnelles, d'après un théorème bien connu de Max Noether, ou encore d'après le théorème de Tsen). La méthode de Salberger peut s'appliquer à d'autres variétés fibrées au-dessus de la droite projective. Ainsi, dans [CT/SwD94], nous avons étudié les conjectures 1 et 2 sur les variétés de dimension quelconque fibrées au-dessus de $\mathbf{P}_{k}^{1}$, la fibre générique étant une variété de SeveriBrauer "généralisée", et dans [CT/Sk/SwD97] nous avons obtenu des résultats sous des hypothèses plus générales sur la fibre générique et les fibres spéciales de la fibration $X \rightarrow \mathbf{P}_{k}^{1}$.

Dans cet article, j'établis une partie des conjectures ci-dessus pour les surfaces réglées $X / C$, c'est-à-dire les surfaces $X$ fibrées en coniques au-dessus d'une courbe $C$ (projective, lisse) de genre quelconque. Par surface fibrée en coniques $X / C$ sur $k$, on entend dans tout l'article une $k$-surface projective, lisse, géométriquement intègre, munie d'un $k$-morphisme dominant $p: X \rightarrow C$ vers une $k$-courbe projective, lisse, géométriquement intègre, morphisme dont toutes les fibres $X_{P}=p^{-1}(P)$ sont des coniques.

Le théorème 1.4 (ci-dessous) est une version relative de la conjecture 1.2 pour une telle $X / C$. Lorsque $C$ est une courbe de genre un, le théorème 1.5 (ci-dessous) établit la conjecture 1.1 pour une telle surface, sous l'hypothèse que le groupe de Tate-Shafarevich de la jacobienne de $C$ est fini (et sous quelques hypothèses parasites). Même sous cette dernière hypothèse, la conjecture 1.2 reste ouverte pour $X$.

Avant de donner les énoncés, fixons quelques notations. Soient $\bar{k}$ une clôture algébrique de $k$ et $\mathcal{G}=\operatorname{Gal}(\bar{k} / k)$. Soit $\bar{X}=X \times_{k} \bar{k}$. Le groupe de Néron-Severi $\mathrm{NS}(\bar{X})$ est ici un $\mathcal{G}$-réseau. Soit $T$ le $k$-tore de groupe des caractères $\hat{T}=\mathrm{NS}(\bar{X})$. Notons $H^{1}(k, T)$ le groupe de cohomologie galoisienne $H^{1}(\mathcal{G}, T(\bar{k}))$. Soit

$$
\mathrm{CH}_{0}(X / C)=\operatorname{Ker}\left[p_{*}: \mathrm{CH}_{0}(X) \rightarrow \mathrm{CH}_{0}(C)\right] .
$$


Les groupes $\mathrm{CH}_{0}(X / C)$ et $H^{1}(k, T)$ sont annulés par 2 (voir $\S 2$ ). On dispose d'une "application caractéristique"

$$
\Phi: \mathrm{CH}_{0}(X / C) \rightarrow H^{1}(k, T) .
$$

Cette application a été définie de diverses manières dans la littérature (cf. [Gros87]). Dans tout l'article, j'utilise la construction d'E. Frossard ([Fro98], §1). Pour chaque place $v \in \Omega$, on a de même une application caractéristique locale $\Phi_{v}: \mathrm{CH}_{0}\left(X_{v} / C_{v}\right)$ $\rightarrow H^{1}\left(k_{v}, T\right)$. Nous pouvons maintenant énoncer les principaux résultats.

Théorème 1.3. Soient $k$ un corps de nombres et $X / C$ une surface fibrée en coniques sur $k$. Supposons que $C$ possède un point $k$-rationnel $P$ à fibre $X_{P}$ lisse. Soit $\alpha \in H^{1}(k, T)$. Si pour chaque place $v$ de $k$, la restriction $\alpha_{v} \in H^{1}\left(k_{v}, T\right)$ est dans l'image de $\Phi_{v}$, alors $\alpha$ est dans l'image de $\Phi$. Plus précisément, si pour chaque place $v$ il existe $z_{v} \in \mathrm{CH}_{0}\left(X_{v} / C_{v}\right)$ tel que $\alpha_{v}=\Phi_{v}\left(z_{v}\right)$, alors il existe $z \in \mathrm{CH}_{0}(X / C)$ tel que $\Phi(z)=\alpha$ et que pour chaque place $v$ la restriction de $z$ dans $\mathrm{CH}_{0}\left(X_{v} / C_{v}\right)$ coincide avec $z_{v}$.

Le groupe $\mathrm{CH}_{0}(X / C)$ est fini, et les groupes $\mathrm{CH}_{0}\left(X_{v} / C_{v}\right)$ sont finis et presque tous nuls ([Gros87], [Fro98]). L'accouplement (1.1) donne naissance à un complexe de groupes abéliens finis, annulés par 2:

$$
\mathrm{CH}_{0}(X / C) \rightarrow \bigoplus_{v \in \Omega} \mathrm{CH}_{0}\left(X_{v} / C_{v}\right) \rightarrow \operatorname{Hom}(\operatorname{Br}(X) / \operatorname{Br}(C), \mathbf{Q} / \mathbf{Z}) .
$$

Théorème 1.4. Soient $k$ un corps de nombres totalement imaginaire et $\Omega$ l'ensemble de ses places. Soit $X / C$ une surface fibrée en coniques sur $k$. Supposons $C(k) \neq \emptyset$. Alors le complexe naturel de groupes abéliens finis (1.5):

$$
\mathrm{CH}_{0}(X / C) \rightarrow \bigoplus_{v \in \Omega} \mathrm{CH}_{0}\left(X_{v} / C_{v}\right) \rightarrow \operatorname{Hom}(\operatorname{Br}(X) / \operatorname{Br}(C), \mathbf{Q} / \mathbf{Z})
$$

est exact.

On comparera les théorèmes 1.3 et 1.4 avec les Cor. (7.3) et Thm. (7.5) de [Sal88], avec les Thm. 6.2 (ii) et Thm. 6.2 (i) de [CT/SwD94], et avec les généralisations obtenues au $\S 4$ de [CT/Sk/SwD97].

Théorème 1.5. Soient $k$ un corps de nombres totalement imaginaire et $X / C$ une surface fibrée en coniques sur $k$. Supposons:

(i) La courbe $C$ est de genre un.

(ii) Le quotient $\operatorname{Br}(X) / p^{*} \operatorname{Br}(C)=0$.

(iii) Le groupe de Tate-Shafarevich de la jacobienne de $C$ est fini.

$S$ 'il existe une famille $\left\{z_{v}\right\}_{v \in \Omega}$ de zéro-cycles de degré un qui est orthogonale au groupe de Brauer de X par l'accouplement (1.1), alors il existe un zéro-cycle de degré un sur $X$. En d'autres termes, l'obstruction de Brauer-Manin à l'existence d'un zéro-cycle de degré un est la seule obstruction.

On comparera le théorème 1.5 avec [Sal88], Thm. (7.6), avec [CT/SwD94], Thm. 5.1 et avec [CT/Sk/SwD97], Thm. 4.1.

Les méthodes ici développées permettent également de donner une bonne borne pour le niveau des corps des fonctions d'une surface réglée définie sur un corps de nombres: sous une hypothèse mineure (Théorème 9.3), si -1 est une somme de carrés dans le corps des fonctions, c'est une somme d'au plus 4 carrés (pour une surface quelconque, la borne est 8). 
Le présent article est à certains égards plus proche de l'article original [Sal88] de Salberger que ne l'étaient les articles [CT/SwD94] et [CT/Sk/SwD97]. Etant donnée une famille de zéro-cycles locaux (sur chaque $X_{v}$ ), il s'agit de trouver un zéro-cycle global ( $\operatorname{sur} X$ ). On transforme le problème en un problème d'approximation de fonctions rationnelles $f_{v}$ sur chaque $C_{v}$ par une fonction rationnelle $f$ sur $C$. L'article diffère néanmoins de [Sal88] en plusieurs points clés.

1) Dans [Sal88], où $C=\mathbf{P}_{k}^{1}$, un argument fondamental est la décomposition canonique des polynômes en une variable (op. cit., §6). Cette décomposition repose sur la division euclidienne dans l'anneau $k[t]$. Cela semblait bloquer les généralisations au cas d'une courbe $C / k$ de genre quelconque. Supposons $C$ munie d'un point $k$-rationnel $P_{\infty}$, notons $U=C \backslash P_{\infty}$ et $k[U]=\Gamma\left(U, \mathcal{O}_{U}\right)$. L'idée nouvelle et fort simple, trouvée à Bombay un soir de Janvier 1996, est que le théorème de Riemann-Roch sur la courbe $C$ permet de trouver, sur $k[U]$, un substitut (non canonique) à la division euclidienne (voir (5.2) ci-dessous). C'est la principale innovation technique du présent article.

2) On a remplacé ici les lemmes de position générale pour les zéro-cycles du $\S 5$ de [Sal88] par des lemmes de déplacement plus faibles mais purement algébriques ( $\S 3$ pour les théorèmes 1.3 et 1.4; appendice d'E. Frossard et V. Suresh [Fr/Su99] pour le théorème 1.5).

3) Par ailleurs, si l'on procède bien par réduction aux zéro-cycles effectifs, on n'a pas besoin, pour les théorèmes 1.3 et 1.4, de préciser le degré de ces zéro-cycles (à la différence de [Sal88], Théorème (3.1)), ce qui sera sans doute utile pour les généralisations.

4) Outre les techniques employées dans la démonstration des théorèmes 1.3 et 1.4, la démonstration du théorème 1.5 utilise la "suite duale" de Cassels-Tate pour la jacobienne de la courbe $C$ (conjecture 1.2 pour $C$ ).

5) D'autres différences sont mentionnées dans le corps de l'article.

On trouvera au $\S 2$ un certain nombre de rappels, qui rendent la lecture du présent article essentiellement indépendante de celle des articles cités dans cette introduction.

\section{Groupe de Pichrd et groupe de Chow DES SURFACES RÉGLÉES (RAPPELS)}

Soient $k$ un corps de caractéristique zéro, $\bar{k}$ une clôture algébrique, $\mathcal{G}=\operatorname{Gal}(\bar{k} / k)$. Soit $C / k$ une courbe projective, lisse, géométriquement intègre, et soit $p: X \rightarrow C$ une surface fibrée en coniques, comme définie dans l'introduction.

Pour $P$ point fermé de $C$, de corps résiduel $k(P)$, la fibre $X_{P}=p^{-1}(P) / k(P)$ est une conique qui, lorsqu'elle n'est pas lisse, est une union de deux droites qui sont définies soit sur $k(P)$, soit sur une extension quadratique de $k(P)$, et qui se coupent transversalement en un unique $k(P)$-point. On note $\left\{P_{i}\right\}_{i \in I}$ l'ensemble des points fermés $P \in C$ dont la fibre $p^{-1}(P)$ n'est pas lisse.

Pour toute extension de corps $L / k$, on note $L(C)$ le corps des fonctions de $C \times{ }_{k} L$. On note $\bar{X}=X \times_{k} \bar{k}$ et $\bar{C}=C \times_{k} \bar{k}$.

On suppose qu'il existe un point $k$-rationnel, noté $P_{\infty} \in C(k)$, dont la fibre $X_{\infty}=X_{P_{\infty}}$ est une conique lisse. On note $U=C \backslash P_{\infty}$.

On note $A / k(C)$ l'algèbre de quaternions correspondant à la fibre générique de $p$. On note $\operatorname{Nrd}\left(A^{*}\right) \subset k(C)^{*}$ le groupe des normes réduites. 
On note $k(C)_{\mathrm{dn}}^{*}$ le sous-groupe de $k(C)^{*}$ formé des fonctions qui en tout point $P \in C$ s'écrivent comme le produit d'une unité en $P$ par un élément de $\operatorname{Nrd}\left(A^{*}\right)$. C'est aussi le groupe des fonctions rationnelles non nulles dont le diviseur sur $C$ est l'image directe d'un zéro-cycle sur $X$ (pour tout ceci, voir [Sal85], [CT/Sko93], $\S 1$, [Fro98], §3). Etant donné un ouvert non vide $W \subset C$, on utilisera la notation $k(W)_{\mathrm{dn}}^{*}$ pour le sous-groupe de $k(C)^{*}$ formé des fonctions qui partout localement sur $W$ s'écrivent comme le produit d'une unité et d'une norme réduite de $A$. Ce

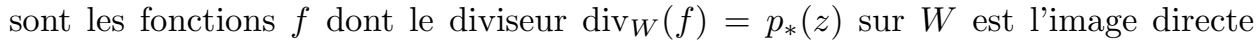
$p_{*}(z)$ d'un zéro-cycle sur $X_{W}=X \times_{C} W$. On a le lemme (voir [CT/Sko93], $\S 1$ et Prop. 2.1):

Lemme 2.1. Soit $W$ une $k$-courbe lisse et géométriquement intègre. Soit $p: X \rightarrow$ $W$ une surface fibrée en coniques, et soit $A / k(W)$ l'algèbre de quaternions associée. Soit $z$ un zéro-cycle sur $X$. Alors les conditions suivantes sont équivalentes:

a) Le zéro-cycle $z$ est rationnellement équivalent à zéro sur $X$.

b) Il existe $h \in A^{*}$ tel que $p_{*}(z)=\operatorname{div}_{W}(\operatorname{Nrd}(h))$.

Ainsi l'application qui à une fonction $f \in k(W)_{\mathrm{dn}}^{*}$ telle que $\operatorname{div}_{W}(f)=p_{*}(z)$ associe la classe de $z$ induit un isomorphisme

$$
k(W)_{\mathrm{dn}}^{*} / k[W]^{*} \operatorname{Nrd}\left(A^{*}\right) \simeq \mathrm{CH}_{0}\left(X_{W} / W\right),
$$

où $k[W]=H^{0}\left(W, \mathcal{O}_{W}\right)$ et $k[W]^{*}$ est le groupe des fonctions inversibles sur $W$ (cf. [CT/Sko93], loc. cit.). En particulier, on a

$$
k(C)_{\mathrm{dn}}^{*} / k^{*} \operatorname{Nrd}\left(A^{*}\right) \simeq \mathrm{CH}_{0}(X / C)
$$

et

$$
k(U)_{\mathrm{dn}}^{*} / k^{*} \operatorname{Nrd}\left(A^{*}\right) \simeq \mathrm{CH}_{0}\left(X_{U} / U\right)
$$

puisque toute fonction inversible sur $U$ est constante. On voit ainsi que les groupes $\mathrm{CH}_{0}(X / C)$ et $\mathrm{CH}_{0}\left(X_{U} / U\right)$ sont annulés par 2 .

On notera $\operatorname{Spec}(E)$ le sous $k$-schéma fermé réduit de $U$ définissant les points $P_{i}, i \in I$, de mauvaise réduction pour $X / C$. On a $E=\prod_{i \in I} k_{i}$, où $k_{i}$ désigne le corps résiduel en $P_{i}$. A chaque point $P_{i}$ est associé une extension $l_{i} / k_{i}$, séparable de degré 2 . Suivant que $l_{i}=k_{i} \times k_{i}$ ou que $l_{i}$ est un corps les deux composantes de la fibre géométrique en $P_{i}$ sont définies sur $k_{i}$ ou sur l'extension quadratique $l_{i}$ (dans le premier cas, la fibration $X / C$ n'est pas relativement minimale). On note $F=\prod_{i \in I} l_{i}$. La flèche naturelle $E=\prod_{i \in I} k_{i} \rightarrow F=\prod_{i \in I} l_{i}$ fait de $F$ une $E$-algèbre finie étale de rang constant 2. On note $N_{i}$ la norme de $l_{i}$ à $k_{i}$, et $N$ la norme de $F$ à $E$.

On dispose d'une application dite de spécialisation

$$
s p: k(U)_{\mathrm{dn}}^{*} \rightarrow E^{*} / N F^{*}=\prod_{i \in I} k_{i}^{*} / N_{i} l_{i}^{*} .
$$

Sur la composante $i$, cette application est ainsi définie: on représente $f \in k(U)_{\mathrm{dn}}^{*}$ comme le produit d'une unité $u_{i} \in \mathcal{O}_{U, P_{i}}^{*}$ dans l'anneau local en $P_{i}$ par une norme réduite de $A$, et on regarde la classe de $u_{i}\left(P_{i}\right) \in k_{i}^{*}$ dans $k_{i}^{*} / N_{i} l_{i}^{*}$. On vérifie que cela ne dépend pas du choix de l'écriture; en particulier cette application est triviale sur $\operatorname{Nrd}\left(A^{*}\right)$. Ceci se voit simplement au niveau du complété de $k(C)$ en $P_{i}$, voir [Sal85] et [Fro98], Définition 4.2. Ceci se voit encore en notant que l'image de $s p_{i}(f) \in k_{i}^{*} / N_{i} l_{i}^{*} \subset \operatorname{Br}\left(k_{i}\right)$ n'est autre que le résidu en $P_{i}$ de $f \cup A \in H^{3}(k(C), \mathbf{Z} / 2)$; ici $f$ est vue comme une classe dans $k(C)^{*} / k(C)^{* 2}=H^{1}(k(C), \mathbf{Z} / 2)$ et $A$ comme 
une classe dans $H^{2}(k(C), \mathbf{Z} / 2)$, qui est la 2-torsion du groupe de Brauer de $k(C)$. Mais pour $f \in \operatorname{Nrd}\left(A^{*}\right)$, on a $f \cup A=0 \in H^{3}(k(C), \mathbf{Z} / 2$ ) (partie facile de la proposition 2.3 ci-dessous).

L'application $s p$ définie en (2.3) induit une application

$$
\widetilde{s p}: k(U)_{\mathrm{dn}}^{*} \rightarrow E^{*} / k^{*} N F^{*},
$$

où $E^{*} / k^{*} N F^{*}$ est le quotient de $E^{*} / N F^{*}=\prod_{i \in I} k_{i}^{*} / N_{i} l_{i}^{*}$ par l'image de l'application diagonale $k^{*} \rightarrow \prod_{i \in I} k_{i}^{*} / N_{i} l_{i}^{*}$. L'application $\widetilde{s p}$ est triviale sur $k^{*} \operatorname{Nrd}\left(A^{*}\right)$. Compte tenu de l'isomorphisme $k(U)_{\mathrm{dn}}^{*} / k^{*} \operatorname{Nrd}\left(A^{*}\right) \simeq \mathrm{CH}_{0}\left(X_{U} / U\right)$, elle induit donc un homomorphisme

$$
\Phi_{U}: \mathrm{CH}_{0}\left(X_{U} / U\right) \rightarrow E^{*} / k^{*} N F^{*} .
$$

Soit $\left[X_{\infty}\right] \in \operatorname{Br}(k)$ la classe de la conique $X_{\infty}$, fibre de $X / C$ au-dessus du point $P_{\infty}$. L'application qui à $f \in k(U)_{\mathrm{dn}}^{*}$ associe l'élément $v_{P_{\infty}}(f)\left[X_{\infty}\right] \in \operatorname{Br}(k)$ est un homomorphisme $k(U)_{\mathrm{dn}}^{*} \rightarrow \operatorname{Br}(k)$ qui s'annule clairement sur $k^{*}$. Comme $v_{P_{\infty}}(f)\left[X_{\infty}\right]$ est le résidu de $f \cup A \in H^{3}(k(C), \mathbf{Z} / 2)$ au point $P_{\infty}$, cet homomorphisme s'annule aussi sur $\operatorname{Nrd}\left(A^{*}\right)$. Composant avec l'isomorphisme

$$
k(U)_{\mathrm{dn}}^{*} / k^{*} \operatorname{Nrd}\left(A^{*}\right) \simeq \mathrm{CH}_{0}\left(X_{U} / U\right),
$$

on obtient un homomorphisme

$$
\mathrm{CH}_{0}\left(X_{U} / U\right) \rightarrow \mathrm{Br}(k)
$$

qui peut encore être décrit de la façon suivante: Etant donné un élément $z \in$ $\mathrm{CH}_{0}\left(X_{U} / U\right)$, on le représente par un zéro-cycle $Z$ sur $X_{U}$, et on associe à $z$ la classe $\operatorname{deg}(Z)\left[X_{\infty}\right] \in \operatorname{Br}(k)$.

Comme $\bar{X} / \bar{C}$ est une fibration en coniques, la composante neutre $\operatorname{Pic}^{\circ}(\bar{C})$ de la variété de Picard de $\bar{C}$ s'envoie isomorphiquement par $p^{*}$ sur la composante neutre $\operatorname{Pic}^{\circ}(\bar{X})$ de la variété de Picard de $\bar{X}$. On dispose donc de la suite exacte de modules galoisiens:

$$
0 \rightarrow \operatorname{Pic}^{o}(\bar{C}) \rightarrow \operatorname{Pic}(\bar{X}) \rightarrow \mathrm{NS}(\bar{X}) \rightarrow 0 .
$$

Le groupe de Néron-Severi géométrique $\operatorname{NS}(\bar{X})$ est un groupe abélien libre de type fini, qui s'insère dans une suite exacte de modules galoisiens:

$$
0 \rightarrow P_{1} \rightarrow P_{2} \oplus \mathbf{Z} f \rightarrow \mathrm{NS}(\bar{X}) \rightarrow \mathbf{Z} \rightarrow 0 .
$$

Ici $P_{1}=\bigoplus \mathbf{Z} x$ est le groupe abélien libre sur les points $x \in \bar{C}$ à fibre singulière, $P_{2} \subset \operatorname{Div}(\bar{X})$ est le groupe abélien libre sur les composantes des fibres géométriques singulières, et $f$ désigne la classe d'une fibre au-dessus du point $k$-rationnel $P_{\infty}$. Dans cette suite, la flèche $\mathrm{NS}(\bar{X}) \rightarrow \mathbf{Z}$ est donnée par la restriction à la fibre générique de $\bar{X} / \bar{C}$, fibre qui est isomorphe à $\mathbf{P}_{\bar{k}(C)}^{1}$ (théorème de Tsen). La flèche $P_{1} \rightarrow P_{2} \oplus \mathbf{Z}$ envoie le générateur $x \in \bar{C}$ sur $p^{-1}(x)-p^{-1}\left(P_{\infty}\right)$. Le module galoisien $\operatorname{NS}(\bar{X})$ est un $\mathcal{G}$-réseau. Soit $T$ le $k$-tore de groupe des caractères $\hat{T}=\operatorname{NS}(\bar{X})$.

De la suite exacte de $\mathcal{G}$-réseaux (2.8) on déduit par antidualité une suite exacte de $k$-tores algébriques:

$$
1 \rightarrow \mathbb{G}_{m, k} \rightarrow T \rightarrow \mathbb{G}_{m, k} \times_{k} R_{F / k} \mathbb{G}_{m, F} \rightarrow R_{E / k} \mathbb{G}_{m, E} \rightarrow 1 .
$$

La cohomologie galoisienne de cette dernière suite et le théorème 90 de Hilbert donnent naissance à une suite exacte:

$$
0 \rightarrow H^{1}(k, T) \rightarrow E^{*} / k^{*} N F^{*} \rightarrow \operatorname{Br}(k) \rightarrow H^{2}(k, T) .
$$


La flèche $E^{*} / k^{*} N F^{*} \rightarrow \operatorname{Br}(k)$ est obtenue par passage au quotient à partir de l'application composée

$$
E^{*} / N F^{*}=\bigoplus_{i \in I} k_{i}^{*} / N_{i} l_{i}^{*} \subset \bigoplus_{i \in I} \operatorname{Br}\left(k_{i}\right) \rightarrow \operatorname{Br}(k),
$$

la dernière flèche étant la somme des applications de corestriction. (Pour une discussion de tout ceci, voir [Fro98].)

Lemme 2.2. On a le diagramme commutatif de suites exactes:

$$
\begin{aligned}
& 0 \rightarrow \mathrm{CH}_{0}(X / C) \rightarrow \mathrm{CH}_{0}\left(X_{U} / U\right) \rightarrow \operatorname{Br}(k) \\
& \downarrow \Phi \quad \downarrow \Phi_{U} \quad \downarrow= \\
& 0 \rightarrow H^{1}(k, T) \quad \rightarrow \quad E^{*} / k^{*} N F^{*} \rightarrow \operatorname{Br}(k) .
\end{aligned}
$$

Dans ce diagramme, $\Phi$ est la flèche (1.4), $\Phi_{U}$ est la flèche (2.5), la flèche de restriction $\mathrm{CH}_{0}(X / C) \rightarrow \mathrm{CH}_{0}\left(X_{U} / U\right)$ est induite par (2.1) et (2.2), la flèche $\mathrm{CH}_{0}\left(X_{U} / U\right) \rightarrow \mathrm{Br}(k)$ est (2.6); enfin la suite inférieure provient de (2.10).

Démonstration. Pour la commutativité de la partie gauche du diagramme, on se reportera à [Sal88], Thm. (4.4), et à [Fro98], Prop. 3.8. La commutativité du diagramme de droite revient à l'énoncé suivant: Pour $f \in k(U)_{\mathrm{dn}}^{*}$, l'image de $\widetilde{s p}(f)$ par l'application $E^{*} / k^{*} N F^{*} \rightarrow \operatorname{Br}(k)$ coïncide avec $v_{P_{\infty}}(f)\left[X_{\infty}\right]$. Cette formule résulte du complexe usuel (loi de réciprocité pour la cohomologie galoisienne des courbes propres et lisses)

$$
H^{3}(k(C), \mathbf{Z} / 2) \rightarrow \bigoplus_{P \in C} H^{2}(k(P), \mathbf{Z} / 2) \rightarrow H^{2}(k, \mathbf{Z} / 2),
$$

où les flèches de gauche sont les flèches de résidu en chaque point fermé $P \in C$, et où celles de droite se traduisent comme les applications de corestriction ${ }_{2} \operatorname{Br}(k(P)) \rightarrow$ ${ }_{2} \operatorname{Br}(k)$, et des faits suivants:

a) Pour $P \in C$ point fermé de bonne réduction pour $X / C$, donc pour $A$, et $f \in k(C)^{*}$, le résidu en $P$ de $f \cup A$ est précisément $v_{P}(f) A(P) \in \operatorname{Br}(k(P))$, qui est nul si et seulement si $f$ est "dn" en $P$. Au point $P_{\infty}$, ce résidu est donc $v_{P_{\infty}}(f)\left[X_{\infty}\right]$.

b) Pour $P$ l'un des $P_{i}$, le résidu de $f \cup A$ est précisément $s p_{i}(f)$.

Rappelons le résultat suivant, cas particulier d'un résultat de Merkur'ev et Suslin ([M/S82], Thm. (12.1)):

Proposition 2.3. Soit $F$ un corps, $\operatorname{car}(F) \neq 2$, soit $A$ une algèbre de quaternions sur $F$ et soit $f \in F^{*}$. Alors $f$ est une norme réduite de $A$ si et seulement si le cup-produit $f \cup A=0 \in H^{3}(F, \mathbf{Z} / 2)$.

Dans cet énoncé, on a noté abusivement $f$ la classe de $f$ dans $F^{*} / F^{* 2}=$ $H^{1}(F, \mathbf{Z} / 2)$ et $A$ la classe de $A$ dans la 2-torsion du groupe de Brauer de $F$, ellemême isomorphe à $H^{2}(F, \mathbf{Z} / 2)$.

Théorème 2.4. Soit $k$ un corps de nombres, et soit $X / C$ une surface fibrée en coniques sur $k$. Pour $v$ place de $k$, notons $X_{v}=X \times \times_{k} k_{v}$ et $C_{v}=C \times{ }_{k} k_{v}$. On a:

(i) Le groupe $\mathrm{CH}_{0}(X / C)$ est fini.

(ii) Les groupes $\mathrm{CH}_{0}\left(X_{v} / C_{v}\right)$ sont finis, et nuls pour presque toute place $v$ de $k$. Supposons qu'il existe $P_{\infty} \in C(k)$ à fibre $X_{\infty}$ lisse. Soit $U=C \backslash P_{\infty}$, et $U_{v}=$ $U \times{ }_{k} k_{v}$.

(iii) Le groupe $\mathrm{CH}_{0}\left(X_{U} / U\right)$ est fini. $k$.

(iv) Les groupes $\mathrm{CH}_{0}\left(X_{U_{v}} / C_{v}\right)$ sont finis, et nuls pour presque toute place $v$ de 
Les énoncés (i) et (ii) sont dus à M. Gros [Gros87] et T. Ōkōchi (voir aussi [Fro98], Thm. 4.7 et Thm. 4.8). Les énoncés (iii) et (iv) en résultent immédiatement, compte tenu du lemme 2.2 (noter que pour presque toute place $v$ de $k$, la classe de $\left[X_{\infty}\right] \in \operatorname{Br}(k)$ s'annule dans $\left.\operatorname{Br}\left(k_{v}\right)\right)$.

\section{Lemmes DE DÉPlaCeMENT ET D'EFFECTIVITÉ DE ZÉRO-CyCles}

Lemme 3.1. Soit $k$ un corps infini, soient $D$ et $C$ des $k$-courbes projectives, lisses, géométriquement intègres, et $f: D \rightarrow C$ un $k$-morphisme fini et plat. Soit $g=g(D)$ le genre de la courbe $D$. Soit $L$ un faisceau inversible sur $D$, de degré au moins égal à $2 g+1$. Soit $S$ un ensemble fini de points fermés de $D$. Il existe alors un diviseur effectif $\Delta$ sur $D$, qui est une section de $L$, a son support étranger à $S$, et est tel que le zéro-cycle $f_{*}(\Delta)$ sur $C$ ait toutes ses multiplicités égales à 1.

Démonstration. La condition que le degré $d$ de $L$ soit au moins égal à $2 g+1$ assure que le faisceau $L$ est très ample. Soit $H=\mathbf{P}\left(\widehat{H^{0}(D, L)}\right)$. C'est un espace projectif de dimension $d-g$. D'après le théorème de Bertini, on peut trouver un ouvert de Zariski de $H$ tel que pour tout point de cet ouvert, le diviseur correspondant de $D$ soit sans facteur multiple.

Soit $P$ un point fermé de $D$. L'espace projectif des sections de $L \otimes \mathcal{O}_{D}(-P)$ est de dimension strictement plus petite que $H=\mathbf{P}\left(\widehat{H^{0}(D, L)}\right)$ (appliquer le théorème de Riemann-Roch sur la clôture algébrique de $k$, on est alors réduit au cas d'un point de degré un).

Soit $U \subset D \times_{C} D$ l'ouvert complémentaire de la diagonale. C'est une variété de dimension un. Soit $Z \subset U \times_{k} H$ le fermé formé des triples $\left\{P_{1}, P_{2}, h\right\}$, avec $P_{1} \in h$ et $P_{2} \in h$. Les fibres de la projection $Z \rightarrow U$ au-dessus d'un point géométrique $\left(P_{1}, P_{2}\right) \in U(\bar{k})$ sont des espaces projectifs de dimension $d-g-2$, comme on voit en appliquant le théorème de Riemann-Roch. Ainsi la dimension de $Z$ est $d-g-1$, et son image par la projection $U \times_{k} H \rightarrow H$ est un ensemble constructible dont l'adhérence a dimension au plus $d-g-1$.

L'énoncé résulte de la combinaison de ces trois remarques.

Lemme 3.2. Soient $k$ un corps parfait infini, $C$ une $k$-courbe projective, lisse et géométriquement intègre, $X$ une $k$-variété projective, lisse et géométriquement intègre, et $p: X \rightarrow C$ un k-morphisme propre et plat. Soit $A$ un point fermé de $X$, et soient $P_{i}, i=1, \cdots, n$, des points fermés de $C$. Soit z un zéro-cycle sur $X$. Il existe alors un entier $r_{0}>0$ (dépendant de $z$ ) tel que pour tout entier $r \geq r_{0}$, il existe un zéro-cycle effectif $z_{r}$ rationnellement équivalent à $z+r A$ sur $X$, et tel que de plus le zéro-cycle $p_{*}\left(z_{r}\right)$ sur $C$ ait son support en dehors des points $P_{i}$ et de $p(A)$, et ait toutes ses multiplicités égales à un.

Démonstration. Fixons un point fermé $R \in X$ tel que $p(R) \neq p(A)$. Par une variante connue des théorèmes de Bertini (voir [Kl/Al79] et [Co80]), on peut trouver une $k$-courbe $D \subset X$ projective, lisse et géométriquement intègre qui contienne chaque point du support de $z$ ainsi que les points $A$ et $R$. L'application $p_{D}: D \rightarrow C$ induite par $p$ est alors finie et plate. Soit $S$ l'ensemble fini des points fermés de $D$ dont l'image par $p_{D}$ est soit $p(A)$ soit l'un des points $P_{i}$. Soit $g=g(D)$ le genre de $D$. En prenant pour $r_{0}$ le plus petit entier supérieur ou égal à $(2 g+1-\operatorname{deg}(z)) / \operatorname{deg}(A)$, l'énoncé résulte immédatement du lemme précédent. 
Dans le cas particulier qui va nous intéresser ici, celui où $X$ est une surface fibrée en coniques relativement minimale au-dessus d'une courbe $C$ de genre $g$, Salberger [Sal85] montre que tout zéro-cycle de degré au moins égal à $N=2 g+$ $(s-3) / 2$ est rationnellement équivalent à un zéro-cycle effectif. Ici $s$ est le nombre de fibres géométriques singulières de la fibration $X / C$, et l'on suppose la fibration non scindée. Le cas $C=\mathbf{P}_{k}^{1}$ de ce théorème, dû à Coray et l'auteur, est utilisé par Salberger dans [Sal88]. Mais cette démonstration ne permet pas a priori de trouver $z$ tel que $p_{*}(z)$ ait toutes ses multiplicités égales à un et soit étranger à des points fermés $P_{i}$ donnés à l'avance. Dans [Sal88], $\S 5$, Salberger résoud ce problème par un argument spécifique aux corps locaux. Il est fait de même dans [CT/SwD94], Lemma 6.2.1. La présente démonstration évite ce problème. Une autre façon de s'en débarrasser, pour $C=\mathbf{P}_{k}^{1}$, a été utilisée dans [CT/SwD94], Remark 5.1.1 (voir aussi [CT/Sk/SwD97], Remark 4.2 (b)), mais elle ne semble pas s'adapter au cas d'une courbe de base $C$ de genre $g>0$. Le résultat suivant, qui sera utilisé au $\S 8$, a été établi par E. Frossard et V. Suresh ([Fr/Su99], appendice au présent article):

Proposition 3.3. Soit $k$ un corps de caractéristique nulle. Soit $p: X \rightarrow C$ une surface fibrée en coniques au-dessus d'une courbe $C$. Soit $S$ un ensemble fini de points fermés de $C$. Il existe un entier naturel $N=N(X, S)$ tel que pour tout corps $K$ contenant $k$ on ait la propriété suivante: tout zéro-cycle sur $X_{K}=X \times_{k} K$ de degré (sur $K$ ) au moins égal à $N$ est rationnellement équivalent, sur $X_{K}$, à un zéro-cycle effectif $z$ tel que $\left(p_{K}\right)_{*}(z)$ ait son support étranger à $S_{K}$, et ait toutes ses multiplicités égales à un.

\section{Approximation de fonctions SUR Un CORPS LOCAL}

On conserve les notations du $\S 2$. En particulier, on note $U=C \backslash P_{\infty}$. On appelle degré d'une fonction $f \in k[U]$ l'opposé de la valuation $v_{P_{\infty}}(f)$. On dit que $f \in k[U]$ est séparable si le diviseur de $f$ sur $U$ a toutes ses multiplicités égales à un. Pour $f \in k[U]$ non nul, $f$ appartient à $k(U)_{\mathrm{dn}}^{*}$ et est séparable si et seulement si $\operatorname{div}_{U}(f)=\sum_{j} R_{j}$ avec $R_{j_{1}} \neq R_{j_{2}}$ pour $j_{1} \neq j_{2}$ et avec $X_{R_{j}}\left(k\left(R_{j}\right)\right) \neq \emptyset$ pour tout $j$.

Lemme 4.1. Soit $k$ un corps local de caractéristique nulle. Soit $f \in k[U] \cap k(U)_{\mathrm{dn}}^{*}$. Supposons que $f$ est séparable sur $U$ et inversible en les points $P_{i}, i \in I$. Soit $z$ un zéro-cycle effectif sur $X_{U}$ tel que $\operatorname{div}_{U}(f)=p_{*}(z)$.

Pour tout $g \in k[U]$ de même degré que $f$ et suffisamment proche de $f$ dans le $k$-vectoriel $W$ de dimension finie formé des $g \in k[U]$ tels que $v_{P_{\infty}}(g) \geq v_{P_{\infty}}(f)$, on $a$ :

(i) $g \in k(U)_{\mathrm{dn}}^{*}$; plus précisément, il existe un zéro-cycle $z_{1}$ effectif sur $X_{U}$ de même degré que $z$, tel que $\operatorname{div}_{U}(g)=p_{*}\left(z_{1}\right)$.

(ii) $s p(f)=s p(g) \in E^{*} / N F^{*}$.

(iii) $g / f$ est une norme réduite de $A$, et $z$ est rationnellement équivalent à $z_{1}$ sur $X$.

Démonstration. Soit $\operatorname{div}_{U}(f)=\sum_{j} R_{j}=p_{*}(z)$ avec $z$ zéro-cycle effectif sur $X_{U}$. Pour tout point $R_{j}$, il existe un point fermé $Q_{j}$ sur $X$ avec $p\left(Q_{j}\right)=R_{j}$ et $k\left(R_{j}\right) \simeq$ $k\left(Q_{j}\right)$. On peut supposer $z=\sum_{j} Q_{j}$. On choisit pour chaque paire $Q_{j}, R_{j}$ une courbe intègre $C_{j} \subset X$ étale au-dessus de $C$ en $Q_{j}$. En utilisant une version convenable du lemme de Krasner et le théorème des fonctions implicites ([Se64], 
Part II, Chapter III, §10), on voit que si $g$ est "très proche" de $f$, alors $\operatorname{div}_{U}(g)=$ $\sum_{j} T_{j}$ avec $T_{j}=p_{*}\left(S_{j}\right)$ et $S_{j}$ point fermé de $C_{j}$ très proche de $Q_{j}$ (tous les points d'indice $j$ ayant même corps résiduel). Ceci établit le point (i), et le point (ii) est clair, puisque $s p_{i}$ envoie $f$ sur la classe dans $k_{i}^{*} / N_{i} l_{i}^{*}$ de l'évaluation de $f$ en $P_{i}$.

Supposons $k$-adique. Soit $O$ l'anneau des entiers de $k$, et soit $\mathcal{C} / O$ un modèle intègre, régulier, propre de la courbe $C / k$ au-dessus de $O$. Le point rationnel $P_{\infty}$ définit une section de $\mathcal{C} / O$; soit $\mathcal{U}$ le complémentaire de cette section (ce n'est pas en général un schéma affine). Soit $B_{y}$ l'anneau local de $\mathcal{X}$ au point générique $y$ d'une composante de la fibre spéciale $Y$ de $\mathcal{X} / O$. Soit $\hat{B}_{y}$ le complété de $B_{y}$, et soit $\hat{K}_{y}$ le corps des fractions de $\hat{B}_{y}$. Par le lemme de Hensel, il existe un entier $n$ tel que tout élément de $1+p^{n} B_{y}$ est un carré dans $\hat{B}_{y}$ (la valuation de $p$ dans $B_{y}$ est strictement positive). Il existe donc un entier $m$ tel que tout élément de la forme $f+p^{m} h$ avec $h$ entier en chaque point générique $y$ de la fibre spéciale diffère de $f$, dans chaque $\hat{K}_{y}$, par un carré.

D'après les parties (i) et (ii), on sait déjà que pour $g \in W$ dans un voisinage ouvert $W_{1}$ de $f$, la classe de $(g / f) \cup A \in H^{3}(k(C), \mathbf{Z} / 2)$ a ses résidus triviaux en chaque point fermé de $C$. L'anneau $O[\mathcal{U}]=H^{0}\left(\mathcal{U}, \mathcal{O}_{\mathcal{U}}\right)$ est contenu dans chaque anneau local $B_{y}$. Pour tout $h \in O[\mathcal{U}]$, on voit donc que $\left(f+p^{m} h\right) / f$ est un carré dans chaque $\hat{K}_{y}$, et donc $\left(\left(f+p^{m} h\right) / f\right) \cup A=0 \in H^{3}\left(\hat{K}_{y}, \mathbf{Z} / 2\right)$. La trace de $f+p^{m} O[\mathcal{U}]$ sur le $k$-vectoriel $W$ de l'énoncé est clairement un ouvert $W_{2}$. Pour $g \in W_{1} \cap W_{2}$, on voit donc que la classe $(g / f) \cup A$, sur $\mathcal{C}$, a tous ses "résidus" triviaux: ici les "résidus" sont pris au sens du complexe de Bloch-Ogus défini par Kato [Kato86]. Il en résulte ([Kato86], Proposition 5.2) que la classe $(g / f) \cup A \in H^{3}(k(C), \mathbf{Z} / 2)$ est nulle (ceci vaut même si $k$ est dyadique), et donc, par la proposition 2.3 , que $g / f$ est une norme réduite de $A$. $\operatorname{Soit}_{\operatorname{div}_{U}}(g)=p_{*}\left(z_{1}\right)$ comme dans (i). Du lemme 2.1 il résulte alors que le zéro-cycle $z-z_{1}$ est rationnellement équivalent à zéro sur $X_{U}$, donc aussi sur $X$ puisqu'il est de degré zéro et que la fibre $X_{\infty}$ est une conique lisse.

Supposons $k$ archimédien. Si $k$ est le corps $\mathbf{C}$ des complexes, l'algèbre $A$ est déployée sur $\mathbf{C}(C)$ (théorème de Tsen), et tout élément de $\mathbf{C}(C)$ est une norme réduite. Supposons que $k$ est le corps $\mathbf{R}$ des réels. Soit $T \subset C(\mathbf{R})$ le complémentaire de $p(X(\mathbf{R}))$. C'est une union $T=\bigcup_{s \in S} T_{s}$ d'intervalles et de cercles. Les points $P_{i}$ qui sont réels n'appartiennent pas à $T$. L'hypothèse $f \in \mathbf{R}(U)_{\mathrm{dn}}^{*}$ implique que pour tout point $P$ de $T$, la valuation $v_{P}(f)$ est paire. Ainsi, sur toute composante connexe $T_{s}$ de $T$, la fonction $f$ a un signe constant $\epsilon_{s}= \pm 1$ là où elle est inversible. Sur chaque composante $T_{s}$, choisissons un point $P_{s}$ où $f$ est inversible. Pour $g$ comme dans l'énoncé et suffisamment proche de $f$, on a déjà vu que $g$ appartient à $\mathbf{R}(U)_{\mathrm{dn}}^{*}$. Le raisonnement ci-dessus s'applique donc à $g$ : tout tel $g$ a un signe constant sur chaque $T_{s}$. Dans un voisinage convenable de $f$, tout tel $g$ est inversible en chaque $P_{s}$ et prend en $P_{s}$ le même signe que $f$. Pour tout tel $g$, la fonction $g / f$ prend donc des valeurs positives sur $T$ là où elle est définie. D'après le théorème de Tsen, l'algèbre de quaternions $A / \mathbf{R}(C)$ peut s'écrire $A=(-1, h)_{\mathbf{R}(C)}$, pour $h \in \mathbf{R}(C)^{*}$ convenable. En dehors d'un nombre fini de points d'indétermination, un point $M \in C(\mathbf{R})$ appartient à $T$ si et seulement si $h(M)<0$. Ainsi $h(M)<0$ implique $(g / f)(M)>0$. L'algèbre de quaternions $(h, g / f)_{\mathbf{R}(C)}$ s'annule donc en presque tout point de $C(\mathbf{R})$. Par un théorème de Witt [Witt37], elle est nulle, donc $g / f$ s'écrit $a^{2}-h b^{2}$, avec $a, b \in \mathbf{R}(C)$ et $g / f$ est une norme réduite de l'algèbre $A=(-1, h)_{\mathbf{R}(C)}$. Le résultat sur $z$ et $z_{1}$ résulte alors comme ci-dessus du lemme 2.1 . 
Lemme 4.2. Soit $O$ l'anneau des entiers d'un corps p-adique $k$ de caractéristique résiduelle différente de 2. Soit $\mathcal{C} / O$ une courbe projective et lisse, $C / k$ la fibre générique. Soit $P_{\infty} \in C(k)$, et $\tilde{P}_{\infty} \subset \mathcal{C}$ son adhérence. Soit $\mathcal{U} \subset \mathcal{C}$ le complémentaire de $\tilde{P}_{\infty}$ et $U \subset C$ le complémentaire de $P_{\infty}$. Soit $A$ une algèbre de quaternions sur $k(C)$. Supposons que le lieu de ramification de $A$ sur $\mathcal{C}$ consiste en des $\tilde{P}_{i}=\operatorname{Spec}\left(O_{i}\right) \subset \mathcal{U}$ finis étales sur $O$, d'intersection vide deux à deux, et supposons que pour chaque $i$ le résidu au point générique $P_{i}$ de $\tilde{P}_{i}$ définisse une extension quadratique non ramifiée de $k_{i}$. Soit $f \in O[\mathcal{U}]$. Supposons que pour chaque $i$ on ait $f\left(P_{i}\right) \in O_{i}^{*}$. Alors $f$ est une norme réduite de $A$.

Démonstration. Comme $C / k$ a bonne réduction, et la caractéristique résiduelle est différente de 2, le groupe de cohomologie non ramifiée $H_{\mathrm{nr}}^{3}(k(C), \mathbf{Z} / 2)$ est nul ([Kato86], Cor. 2.9). Il suffit donc de montrer que $f \cup A \in H^{3}(k(C), \mathbf{Z} / 2)$ est partout non ramifié. Par la loi de réciprocité, il suffit de le montrer aux points de $U$. En un point fermé non zéro de $f$ et distinct des $P_{i}$, c'est évident. En un point $P_{i}$, le résidu de $f \cup A$ est la classe de $f\left(P_{i}\right)$ dans $k_{i}^{*} / N_{i} l_{i}^{*} \subset{ }_{2} \operatorname{Br}\left(k_{i}\right)$. Comme $l_{i} / k_{i}$ est non ramifiée, $f\left(P_{i}\right) \in O_{i}^{*}$ a une classe triviale dans $k_{i}^{*} / N_{i} l_{i}^{*}$. L'hypothèse $f\left(P_{i}\right) \in O_{i}^{*}$ assure par ailleurs que le lieu des zéros de $f$ sur $\mathcal{U}$ ne rencontre pas $\tilde{P}_{i}$. Soit alors $P \in U$ un point fermé appartenant à $f=0$, et soit $\tilde{P}$ son adhérence dans $\mathcal{C}$. Alors $\tilde{P}$ ne rencontre aucun des $\tilde{P}_{i}$. Ainsi l'algèbre $A$ est d'Azumaya dans un voisinage de $\tilde{P}$. Du coup $A(P)=0$ car c'est la restriction dans $\operatorname{Br}(k(P))$ d'un élément du groupe de Brauer de l'anneau des entiers de $k(P)$. Le résidu en $P$ de $f \cup A$ est donc nul (il est égal à $A(P)$ multiplié par la valuation de $f$ en $P$ ).

Remarques. (a) Salberger [Sal88], dans son lemme 5.5 (où $U=\mathbf{A}_{k}^{1}$ ), suppose que le polynôme $f(t) \in O[t]$ est unitaire. Ici, cela correspondrait à choisir $f \in O[\mathcal{U}]$ tel que $O[\mathcal{U}] / f$ soit fini sur $O$. Je ne vois nulle part dans la démonstration un point où ceci est nécessaire.

(b) Salberger suppose que l'algèbre $A$ s'annule en $P_{\infty}$. Cette condition est impliquée par nos hypothèses: la section à l'infini est dans l'ouvert de $\mathcal{C}$ où $A$ est Azumaya.

(c) Notre hypothèse implique que sur $\mathcal{C}$ l'algèbre $A$ n'est pas ramifiée au point générique de la fibre spéciale.

\section{Approximation de fonctions sur un CORPs GLOBAL}

On conserve les notations et hypothèses du $\S 2$. On suppose ici en outre que $k$ est un corps de nombres. On considère un modèle projectif et lisse $\mathcal{C} / O$ de $C$ sur un ouvert $\operatorname{Spec}(O)$ de l'anneau des entiers d'un corps de nombres $k$, et le modèle $\mathcal{U}$ de $U$ associé, c'est-à-dire le complémentaire de la section à l'infini de $\mathcal{C}$ définie par l'adhérence du point $P_{\infty}$ dans $\mathcal{C}$. Pour $i \in I$, soit $O_{i}$ l'anneau des $O$-entiers de $k_{i}$. Soit $A \in \operatorname{Br}(k(C))$ la classe de l'algèbre de quaternions associée à la fibration en coniques $X \rightarrow C$, et soient $P_{i}$ les points fermés de $U$ où elle est ramifiée. Soit $\tilde{P}_{i} \subset \mathcal{C}$ l'adhérence de $P_{i}$. Quitte à restreindre $O$, on peut supposer que la réunion des $\tilde{P}_{i}=\operatorname{Spec}\left(O_{i}\right)$ est dans $\mathcal{U}$, qu'elle est finie et étale sur $O$, et que pour chaque $i$ le résidu au point générique $P_{i}$ de $\tilde{P}_{i}$ définit une extension quadratique non ramifiée de $k_{i}$.

On utilise la notion de degré sur $k[U]$ définie plus haut, à savoir l'opposé de la valuation en $P_{\infty}$. Soit $r$ la somme des degrés (sur $k$ ) des points fermés $P_{i}$. Soit $g$ le genre de $C$. 
Lemme 5.1. Pour tout entier $n>2 g-2+r$, le sous-espace $V_{n}$ de $k[U]$ formé des fonctions de degré au plus $n$ s'envoie surjectivement sur $\bigoplus_{i} k_{i}$.

Démonstration. Pour tout entier $n>0$, on a la suite exacte de $\mathcal{O}_{C}$-modules cohérents:

$$
0 \rightarrow \mathcal{O}_{C}\left(n P_{\infty}-\sum_{i \in I} P_{i}\right) \rightarrow \mathcal{O}_{C}\left(n P_{\infty}\right) \rightarrow \bigoplus_{i \in I} k_{i} \rightarrow 0 .
$$

Pour $n>2 g-2+r$, on a $H^{1}\left(C, \mathcal{O}_{C}\left(n P_{\infty}-\sum_{i} P_{i}\right)\right)=0$ par dualité de Serre. L'application $H^{0}\left(C, \mathcal{O}_{C}\left(n P_{\infty}\right)\right) \rightarrow \oplus_{i \in I} k_{i}$ est donc surjective pour tout tel $n$.

Dans la suite, on fixe un entier $n$ tel que $n>2 g-2+r$. On fixe une section $k$-linéaire $\sigma=\bigoplus_{i} \sigma_{i}$ de la projection $V_{n} \rightarrow \bigoplus_{i} k_{i}$. Notons $W_{n}$ le noyau de cette projection. On a la décomposition

$$
V_{n}=W_{n} \oplus\left(\bigoplus_{i \in I} \sigma_{i}\left(k_{i}\right)\right) .
$$

Cette décomposition est invariante par changement de corps de base. Pour chacun des $k$-vectoriels $W_{n}$ et $k_{i}$, choisissons une base. Quitte à restreindre l'ouvert $\operatorname{Spec}(O)$, on peut:

(i) trouver une base de $W_{n}$ formée d'éléments de $O[\mathcal{U}]$;

(ii) supposer que chaque $O_{i}$ est libre sur $O$, et l'on fixe alors une $O$-base de $O_{i}$;

(iii) supposer que les images par $\sigma_{i}$ de cette base appartiennent à $O[\mathcal{U}]$.

Lemme principal 5.2. Soient $k$ un corps de nombres et $X, C, U$ comme ci-dessus. Soit $\eta \in E^{*} / N_{E / F} F^{*}$. Soit $n$ comme ci-dessus. Supposons que pour toute place $v$ il existe un élément $f_{v}$ de $V_{n} \otimes_{k} k_{v} \subset k_{v}[U]$ de degré exactement $n$, qui soit dans $k_{v}(U)_{\mathrm{dn}}^{*}$, soit $\operatorname{div}_{U}\left(f_{v}\right)=p_{*}\left(z_{v}\right)$, que le diviseur $\operatorname{div}_{U}\left(f_{v}\right)$ ait toutes ses multiplicités égales à 1 et soit étranger aux points de mauvaise réduction de $X / C$, et qu'enfin $s p_{v}\left(f_{v}\right) \in E_{v}^{*} / N F_{v}^{*}$ soit l'image de $\eta$.

Alors il existe un élément $f \in V_{n}$, de degré $n$, appartenant à $k(U)_{\mathrm{dn}}^{*}$, soit $\operatorname{div}_{U}(f)=p_{*}(z)$, tel que $s p(f)=\eta$. De plus, si $S_{0}$ est un ensemble fini de places de $k$, on peut choisir $f \in V_{n} \subset k[U]$ et $z$ tels que $f$ soit arbitrairement proche de chaque $f_{v} \in V_{n} \otimes_{k} k_{v}$ pour $v \in S_{0}$, et que pour toute place $v$, les cycles $z$ et $z_{v}$ soient rationnellement équivalents sur $X_{v}$.

Démonstration. On note $S$ un ensemble fini de places contenant:

(a) les places archimédiennes;

(b) les places dyadiques;

(c) les places finies $v$ telles que l'image de $\eta$ dans $E_{v}^{*} / N F_{v}^{*}$ soit non triviale;

(d) les places finies $v$ pour lesquelles $\mathrm{CH}_{0}\left(X_{v} / C_{v}\right) \neq 0$;

(e) les places finies non dans $\operatorname{Spec}(O)$ (avec $O$ comme ci-dessus);

(f) les places de $S_{0}$.

Soit $S_{1}$ l'ensemble des places telles que $A_{v}=A \otimes_{k(C)} k_{v}(C)$ soit triviale dans $\operatorname{Br}\left(k_{v}(C)\right)$. Par le théorème de Tsen, il existe une extension finie $K / k$ telle que $A$ s'annule dans $\operatorname{Br}(K(C))$. Par le théorème de Tchebotarev, il existe une infinité de places $v$ de $k$ totalement décomposées dans $K$. Ainsi $S_{1}$ est infini. Observons que pour toute place $v \in S_{1}$, et tout $i$, l'extension quadratique $l_{i, v} / k_{i, v}$ est triviale. 
Chaque $f_{v} \in V_{n, v}=V_{n} \otimes_{k} k_{v}$ s'écrit de façon unique:

$$
f_{v}=g_{v}+\sum_{i} \sigma_{i}\left(\rho_{i, v}\right)
$$

avec $g_{v} \in W_{n, v}$ et $\rho_{i, v} \in k_{i, v}^{*}=\left(k_{i} \otimes_{k} k_{v}\right)^{*}$.

Par approximation forte (on utilise ici le fait que $S_{1} \backslash S$ est non vide), il existe $g \in W_{n}$, de coefficients (par rapport à la base fixée) appartenant à $O_{S \cup S_{1}}$ (l'anneau des entiers en-dehors de $S \cup S_{1}$ ), et très proche des coefficients de $f_{v}$ pour $v \in S$.

Comme $S_{1} \backslash S$ est infini, le théorème de Dirichlet généralisé ([San82], Cor. 4.4; cette variante due à Sansuc et l'auteur est une conséquence d'un théorème de Waldschmidt) permet de trouver, pour chaque $i$, un élément $\rho_{i} \in k_{i}^{*}$ qui soit très proche de $\rho_{i, v}$ pour $v \in S$ et qui soit une unité de $k_{i}$ en dehors de $S \cup S_{1} \cup w_{i}$, où $w_{i}$ est une place (finie) de $k_{i}$ étrangère à $S \cup S_{1}$ et telle que de plus $w_{i}\left(\rho_{i}\right)=1$.

On trouve ainsi un $f \in V_{n}$, appartenant à $O[\mathcal{U}]$, très proche de $f_{v}$ aux places de $S$, et satisfaisant $f\left(P_{i}\right)=\rho_{i}$.

Montrons que pour toute place $w \neq w_{i}$ de $k_{i}, \eta_{i} \in k_{i}^{*} / N_{i} l_{i}^{*}$ et $f\left(P_{i}\right)=\rho_{i} \in k_{i}^{*}$ ont même image dans $k_{i, w}^{*} / N_{i} l_{i, w}^{*}$.

Pour $w$ au-dessus d'une place $v$ de $S_{1}$, c'est clair, car $k_{i, v}^{*} / N_{i} l_{i, v}^{*}=1$.

Pour $w$ au-dessus d'une place de $S$, cela résulte du fait que $f$ est très proche de $f_{v}$, donc $f\left(P_{i}\right)$ et $f_{v}\left(P_{i}\right)$ ont même image dans $k_{i, v}^{*} / N_{i} l_{i, v}^{*}$, et par hypothèse l'image de $f_{v}\left(P_{i}\right)$ dans $k_{i, v}^{*} / N_{i} l_{i, v}^{*}$ est égale à celle de $\eta_{i}$.

Soit $w$ une place de $k_{i}$ au-dessus d'une place non dans $S$. L'extension $l_{i, w} / k_{i, w}$ est non ramifiée. Pour une telle place $w \neq w_{i}$, la classe de $\rho_{i} \in k_{i}^{*}$ est une $w$-unité, donc $\rho_{i}$ a une image triviale dans $k_{i, w}^{*} / N_{i} l_{i, w}^{*}$. Par définition de $S, \eta_{i}$ a une image triviale dans $k_{i, w}^{*} / N_{i} l_{i, w}^{*}$.

Ainsi les classes de $f\left(P_{i}\right)$ et $\eta_{i}$ dans $k_{i}^{*} / N_{i} l_{i}^{*}$ coïncident dans $k_{i, w}^{*} / N_{i} l_{i, w}^{*}$ pour toute place $w$ de $k_{i}$, sauf peut-être la place $w_{i}$. Par la loi de réciprocité de la théorie du corps de classes global, elles coïncident aussi en cette place, et elles cö̈ncident dans $k_{i}^{*} / N_{i} l_{i}^{*}$.

Nous avons donc établi l'assertion $s p(f)=\eta$.

La démonstration ci-dessus montre qu'en la place $w_{i}$, l'extension $l_{i} / k_{i}$ est décomposée: en effet d'une part $w_{i}\left(\rho_{i}\right)=1$, d'autre part $\rho_{i}$ est une norme locale en $w_{i}$, puisque sa classe dans $k_{i, w_{i}}^{*} / N_{i} l_{i, w_{i}}^{*}$ coïncide avec celle de $f\left(P_{i}\right)$, qui est triviale.

Par construction, $f$ est très proche de $f_{v}$ pour toute place $v \in S$. Il reste à montrer que $f$ appartient à $k(U)_{\mathrm{dn}}^{*}$.

Pour $v \in S$, d'après le lemme 4.1 , on a $f \in k_{v}(U)_{\mathrm{dn}}^{*}$, plus précisément $f / f_{v} \in$ $\operatorname{Nrd}\left(A_{v}\right)$, et $\operatorname{div}_{U}(f)=p_{*}\left(z_{v}\right)$ avec $z_{v}$ rationnellement équivalent à $z \operatorname{sur} X_{v}$.

Pour $v \in S_{1}$, comme $A$ est déployée sur $k_{v}(C)$, on a certainement $f \in \operatorname{Nrd}\left(A_{v}\right)$, donc a fortiori $f \in k_{v}(U)_{\mathrm{dn}}^{*}$.

Pour $v \notin S \cup S_{1}$ et $v$ non au-dessous d'une place $w_{i}$, le lemme 4.2 assure $f \in$ $\operatorname{Nrd}\left(A_{v}\right)$.

Ceci vaut encore pour les places $v$ au-dessous d'une place $w_{i}$. On a vu en effet que $w_{i}$ est décomposée dans $l_{i} / k_{i}$. Plaçons-nous sur $k_{v}$. Le lieu de ramification $\Delta$ de $A_{v} \operatorname{sur} \mathcal{C} \times_{O} O_{v}$ est situé dans la réunion des $\tilde{P}_{i} \times_{O} O_{v}=\operatorname{Spec}\left(O_{i} \otimes_{O} O_{v}\right)=$ $\bigcup_{w \mid v} \operatorname{Spec}\left(O_{i, w}\right)$. Comme $w_{i}$ est décomposée dans l'extension $l_{i} / k_{i}, \Delta$ est en fait inclus dans la réunion pour tout $i$ des $\bigcup_{w \mid v, w \neq w_{i}} \operatorname{Spec}\left(O_{i, w}\right)$. Mais alors on est dans les conditions d'application du lemme 4.2, car pour $w \mid v$ et $w \neq w_{i}$, on a $f\left(P_{i}\right)=\rho_{i} \in O_{i, w}^{*}$. 
La condition $f \in k(U)_{\mathrm{dn}}^{*}$ se traduit ainsi: pour tout point $P \in U$ différent de l'un des $P_{i}$, le résidu $\delta_{P}(f \cup A) \in H^{2}(k(P), \mathbf{Z} / 2)$ est nul (cette condition est automatiquement vérifiée si $A(P)=0)$. Comme on a montré $f \in k_{v}(U)_{\mathrm{dn}}^{*}$ pour toute place $v$ de $k$, on conclut que pour tout point fermé $P$ comme ci-dessus, le résidu $\delta_{P}(f \cup A) \in H^{2}(k(P), \mathbf{Z} / 2)$ a une image nulle dans $H^{2}\left(k(P)_{w}, \mathbf{Z} / 2\right)$ pour toute place $w$ de $k(P)$. Il est donc nul d'après le principe de Hasse pour le groupe de Brauer, et on a bien $f \in k(U)_{\mathrm{dn}}^{*}$.

Remarques. (a) Chez Salberger, il y a une utilisation du théorème de Dirichlet généralisé pour le coefficient "de plus haut degré" en $t$. Ceci ne semble pas requis.

(b) Dans la démonstration ci-dessus, on n'a utilisé l'existence de fonctions $f_{v}$ que pour $v$ parcourant l'ensemble fini de places décrit au début de la démonstration.

\section{DÉmonstration DU ThÉORÈme 1.3}

On conserve les notations et hypothèses des $\S \S 2$ et 5 . En particulier $k$ est un corps de nombres et $\Omega$ l'ensemble de ses places. On comparera l'énoncé suivant avec [Sal88], Cor. 3.3.

Proposition 6.1. Soit $X / C$ une fibration en coniques. Soit $\varepsilon \in E^{*} / k^{*} N F^{*}$ dans le noyau de l'application composée

$$
E^{*} / k^{*} N F^{*} \rightarrow \operatorname{Br}(k) \rightarrow \operatorname{Br}\left(k\left(X_{\infty}\right)\right) .
$$

Supposons que $\varepsilon_{v}=\varepsilon \times_{k} k_{v}$ appartient à l'image de $\Phi_{U_{v}}$ pour chaque $v \in \Omega$, soit $\varepsilon_{v}=\Phi_{U_{v}}\left(Z_{v}\right)$.

Alors pour tout ensemble fini de places $T \subset \Omega$ et tout entier $n>0$, il existe pour $v \in T$ des zéro-cycles effectifs $z_{v}$ sur $X_{U_{v}}$, tous de même degré supérieur ou égal à $n$, tels que:

a) $z_{v}$ est rationnellement équivalent à $Z_{v}$ sur $X_{U_{v}}$;

b) chaque $p_{*}\left(z_{v}\right)$ a ses multiplicités égales à 1 et est étranger aux points $P_{i}$ de mauvaise réduction pour $X / C$.

Lorsque $\varepsilon \in E^{*} / k^{*} N F^{*}$ est dans le noyau de l'application $E^{*} / k^{*} N F^{*} \rightarrow \operatorname{Br}(k)$, le même énoncé vaut en remplaçant $T$ par l'ensemble $\Omega$ de toutes les places de $k$.

Démonstration. Par hypothèse, on a $\varepsilon_{v}=\Phi_{U_{v}}\left(Z_{v}\right)$ pour $Z_{v}$ un zéro-cycle de $X_{U_{v}}$ dont la classe appartient à $\mathrm{CH}_{0}\left(X_{U_{v}} / U_{v}\right)$.

Soit $\Sigma$ l'ensemble fini ou vide des places de $k$ telles que $X_{\infty}\left(k_{v}\right)=\emptyset$. Montrons que les degrés des $Z_{v}$ pour $v \in \Sigma$ ont tous la même parité. Détaillons ce point non entièrement explicité dans [Sal88]. Par hypothèse, l'image de $\varepsilon$ par l'application $E^{*} / k^{*} N F^{*} \rightarrow \operatorname{Br}(k)$ s'annule dans $\operatorname{Br}\left(k\left(X_{\infty}\right)\right)$, cette image vaut donc soit 0 (premier cas) soit $\left[X_{\infty}\right]$ (second cas). Ces deux cas sont confondus lorsque $\left[X_{\infty}\right]=0$, i.e. lorsque $X_{\infty}(k) \neq \emptyset$, i.e. lorsque $\Sigma=\emptyset$.

Dans le premier cas, l'image de $\varepsilon_{v}$ dans $\operatorname{Br}\left(k_{v}\right)$ est nulle pour tout $v \in \Sigma$ et par le lemme 2.2, le degré de $Z_{v}$ est pair (puisque $\left[X_{\infty}\right]_{v} \neq 0$ pour $v \in \Sigma$ ).

Dans le second cas, l'image de $\varepsilon_{v}$ dans $\operatorname{Br}\left(k_{v}\right)$ vaut $\left[X_{\infty}\right]_{v} \neq 0$ pour tout $v \in \Sigma$, et par le même lemme 2.2, le degré de $Z_{v}$ est impair pour tout $v \in \Sigma$.

Soit $P_{v}$ un point fermé de degré minimum sur $X_{\infty, v}$.

Pour toute place $v \in \Omega$, par application du lemme 3.2 sur le corps de base $k_{v}$, on voit que pour $r_{v}$ entier suffisamment grand, le zéro-cycle $Z_{v}+r_{v} P_{v}$ est rationnellement équivalent à un zéro-cycle $z_{v}$ du type voulu. Pour $v \in \Sigma$, le degré 
de $z_{v}$ est pair dans le premier cas, impair dans le second cas. Pour $v \notin \Sigma$, on peut donner à $z_{v}$ une parité quelconque.

Pour tout ensemble fini $T$ de places de $k$, la première partie de la proposition est établie.

Supposons qu'on soit dans le premier cas, i.e. $\varepsilon$ est dans le noyau de $E^{*} / k^{*} N F^{*} \rightarrow$ $\operatorname{Br}(k)$. Pour tout ensemble fini $T$ de places, on sait qu'on peut trouver des $z_{v}$ du type voulu, pourvu que leur degré soit pair et suffisamment grand. Soit $P$ un point fermé de $X_{\infty}$ de degré pair. Soit $D \subset X$ une courbe projective, lisse, géométriquement intègre, finie sur $C$, contenant le point $P$. D'après le lemme 3.1, pour tout entier $n$ suffisamment grand, le zéro-cycle $n P$ est, sur $D$, rationnellement équivalent à un zéro-cycle effectif $w_{n}$ tel que $p_{*}\left(w_{n}\right)$ ait toutes ses multiplicités égales à 1 et que son support soit étranger aux $P_{i}$ et à $\infty$. Pour presque toute place $v$, on a $\mathrm{CH}_{0}\left(X_{U_{v}} / U_{v}\right)=0$. On peut donc pour un tel $v$ supposer $Z_{v}=0$, et prendre $z_{v}=w_{n, v}$. La seconde partie de l'énoncé est alors claire.

Remarque. Le premier cas, i.e. le cas où $\varepsilon \in E^{*} / k^{*} N F^{*}$ est dans le noyau de l'application $E^{*} / k^{*} N F^{*} \rightarrow \operatorname{Br}(k)$ (il en est ainsi trivialement lorsque $X_{\infty}(k) \neq \emptyset$ ), servira seul dans la suite.

Nous pouvons maintenant établir le théorème 1.3 de l'introduction.

Rappelons que les groupes $\mathrm{CH}_{0}(X / C)$ et $\mathrm{CH}_{0}\left(X_{v} / C_{v}\right)$ sont finis, que ces derniers sont nuls pour presque toute place $v$, et qu'il en est de même des groupes $\mathrm{CH}_{0}\left(X_{U} / U\right)$ et $\mathrm{CH}_{0}\left(X_{U_{v}} / U_{v}\right)$ (thm. 2.4).

Supposons donné pour chaque place $v \in \Omega$ un zéro-cycle $z_{v}$ de degré zéro sur $X_{v}$, dont la classe appartient à $\mathrm{CH}_{0}\left(X_{v} / C_{v}\right)$. On a l'application naturelle $\Phi_{v}$ : $\mathrm{CH}_{0}\left(X_{v} / C_{v}\right) \rightarrow H^{1}\left(k_{v}, T\right)$. Par hypothèse, la famille des $\Phi_{v}\left(z_{v}\right)$ provient d'une classe $\varepsilon \in H^{1}(k, T) \subset E^{*} / k^{*} N F^{*}$. Compte tenu des rappels faits au $\S 2$, cette classe est dans le noyau de la flèche $E^{*} / k^{*} N F^{*} \rightarrow \operatorname{Br}(k)$, et l'on voit que la famille des restrictions $z_{v, U}$ des $z_{v}$ aux $X_{U_{v}}$ satisfait l'hypothèse de la proposition 6.1.

D'après cette proposition, on peut trouver des zéro-cycles effectifs $\zeta_{v}$ sur $X_{U_{v}}$, dont la classe cö̈ncide avec celle de $z_{v, U} \in \mathrm{CH}_{0}\left(X_{U_{v}} / U_{v}\right)$, tous de même degré $n$, qu'on peut prendre supérieur ou égal à $2 g-2+r$ (notations comme au lemme 5.1).

De plus les $p_{*}\left(\zeta_{v}\right)$ sont représentés par des $g_{v} \in k_{v}[U]$, tous de degré $n$, appartenant à $k_{v}[U] \cap k_{v}(U)_{\mathrm{dn}}$, d'image $\varepsilon_{v}$ par l'application $\Phi_{U_{v}}$, et tels que $\operatorname{div}_{U}\left(g_{v}\right)$ soit étranger aux $P_{i}$ et ait toutes ses multiplicités égales à 1 .

On relève alors $\varepsilon$ en $\eta \in E^{*} / N F^{*}$. Quitte à multiplier chaque $g_{v}$ par un scalaire dans $k_{v}^{*}$, on peut supposer que l'image de $g_{v}$ dans $E_{v}^{*} / N F_{v}^{*}$ coïncide avec celle de $\varepsilon$ par l'application évidente $E^{*} / N F^{*} \rightarrow E_{v}^{*} / N F_{v}^{*}$.

En utilisant le lemme principal 5.2, on trouve un élément $g \in k[U]$ appartenant à $k(U)_{\mathrm{dn}}^{*}$, de degré $n$, tel que $\operatorname{div}_{U}(g)=p_{*}(\zeta)$ avec $\zeta$ zéro-cycle effectif sur $X_{U}$, rationnellement équivalent à $\zeta_{v}$ dans $\mathrm{CH}_{0}\left(X_{v}\right)$ pour toute place $v$ et tel que de plus $s p(g)=\eta$.

Observons que $\Phi(g)=\varepsilon \in E^{*} / k^{*} N F^{*}$ appartient au noyau de l'application

$$
E^{*} / k^{*} N F^{*} \rightarrow \operatorname{Br}(k) .
$$

D'après le lemme 2.2, ceci implique que $\zeta$ provient d'une classe $z$ dans $\mathrm{CH}_{0}(X / C)$. On voit alors que pour toute place $v$ on a $z-z_{v}=0 \in \mathrm{CH}_{0}\left(X_{v} / C_{v}\right) \subset \mathrm{CH}_{0}\left(X_{v}\right)$. De plus $\Phi(z)=\varepsilon \in H^{1}(k, T)$.

Ceci établit le théorème 1.3. 


\section{DÉMONSTRATION DU THÉORÈME 1.4}

Soient $k$ un corps de caractéristique zéro et $X / C$ une surface fibrée en coniques sur $k$. Supposons d'abord qu'il existe un point $k$-rationnel $P_{\infty} \in C(k)$ à fibre $X_{\infty}$ lisse. Conservons les notations du $§ 2$. La cohomologie galoisienne de la suite (2.7) donne naissance à la suite exacte:

$$
H^{1}\left(k, \operatorname{Pic}^{o}(\bar{C})\right) \rightarrow H^{1}(k, \operatorname{Pic}(\bar{X})) \rightarrow H^{1}(k, \operatorname{NS}(\bar{X})) \rightarrow H^{2}\left(k, \operatorname{Pic}^{o}(\bar{C})\right) .
$$

Comme $\bar{C}$, resp. $\bar{X}$, est régulier, le groupe de Brauer de $\bar{C}$, resp. de $\bar{X}$, s'injecte dans le groupe de Brauer de $\bar{k}(C)$, resp. de la fibre générique de $\bar{X} / \bar{C}$, laquelle est isomorphe à $\mathbf{P}_{\bar{k}(C)}^{1}$. On a $\operatorname{Br}\left(\mathbf{P}_{\bar{k}(C)}^{1}\right)=\operatorname{Br}(\bar{k}(C))$, et ce dernier groupe est nul par le théorème de Tsen. Ainsi $\operatorname{Br}(\bar{C})=0$ et $\operatorname{Br}(\bar{X})=0$. Le groupe $\operatorname{Br}_{1}(X)=$ $\operatorname{Ker}[\operatorname{Br}(X) \rightarrow \operatorname{Br}(\bar{X})]$ coïncide donc ici avec $\operatorname{Br}(X)$. La suite spectrale de Leray pour la cohomologie étale fournit dans ce cas la suite exacte

$$
\operatorname{Br}(k) \rightarrow \operatorname{Br}(X) \rightarrow H^{1}(k, \operatorname{Pic}(\bar{X})) \rightarrow H^{3}\left(k, \mathbf{G}_{m}\right) \rightarrow H_{\text {ét }}^{3}\left(X, \mathbf{G}_{m}\right)
$$

(en général on aurait $\operatorname{Br}_{1}(X)$ au lieu de $\operatorname{Br}(X)$ ), une suite exacte compatible

$$
0 \rightarrow \operatorname{Br}(k) \rightarrow \operatorname{Br}(C) \rightarrow H^{1}(k, \operatorname{Pic}(\bar{C})) \rightarrow 0
$$

et, compte tenu de $\operatorname{Br}(\bar{C})=0$ et $C(k) \neq \emptyset$, des isomorphismes

$$
\operatorname{Br}(C) / \operatorname{Br}(k)=H^{1}(k, \operatorname{Pic}(\bar{C}))=H^{1}\left(k, \operatorname{Pic}^{o}(\bar{C})\right) .
$$

Pour toute variété abélienne $A$ sur un corps local $k$ non réel, on a $H^{2}(k, A)=0$ (Tate, voir [Milne86], Thm. 3.2, p.51). Le rapporteur signale la démonstration élégante suivante. Supposons $k$ extension finie d'un corps $\mathbf{Q}_{p}$. Notons $A_{\text {tors }}$ le sous-groupe de torsion de $A(\bar{k})$. On a $H^{2}\left(k, A_{\text {tors }}\right) \simeq H^{2}(k, A)$. Soit $T(A)$ le module de Tate de $A(\bar{k})$. Par la dualité locale pour les coefficients finis, le groupe $H^{2}\left(k, A_{\text {tors }}\right)$ est dual du groupe des invariants de $T(A)$ sous $\operatorname{Gal}(\bar{k} / k)$. Ce groupe d'invariants est nul, car il est égal au module de Tate de $A(k)$; or le sous-groupe de torsion de $A(k)$ est fini. Pour toute variété abélienne $A$ sur un corps de nombres $k$ totalement imaginaire, on a aussi $H^{2}(k, A)=0$ (Tate, voir [Milne86], Cor. 6.24, p.111).

On obtient ainsi le:

Lemme 7.1. Soit $X / C$ une surface fibrée en coniques. Supposons qu'il existe un point $k$-rationnel de $C$ à fibre lisse. On a une injection $\operatorname{Br}(X) / \operatorname{Br}(C) \hookrightarrow$ $H^{1}(k, \mathrm{NS}(\bar{X}))$. Si $H^{3}\left(k, \mathbf{G}_{m}\right)$ s'injecte dans $H_{e ́ t}^{3}\left(X, \mathbf{G}_{m}\right)$, par exemple si $H^{3}\left(k, \mathbf{G}_{m}\right)$ $=0$ (il en est ainsi si $k$ est un corps local ou un corps de nombres) ou si $X(k) \neq \emptyset$, alors on a une suite exacte:

$$
0 \rightarrow \operatorname{Br}(X) / \operatorname{Br}(C) \rightarrow H^{1}(k, \operatorname{NS}(\bar{X})) \rightarrow H^{2}\left(k, \operatorname{Pic}^{o}(\bar{C})\right) .
$$

Si $k$ est un corps local non réel, ou un corps de nombres totalement imaginaire, alors on a un isomorphisme $\operatorname{Br}(X) / \operatorname{Br}(C) \simeq H^{1}(k, \operatorname{NS}(\bar{X}))$.

(On note ici systématiquement $\operatorname{Br}(X) / \operatorname{Br}(C)$ le groupe que l'on devrait noter $\left.\operatorname{Br}(X) / p^{*}(\operatorname{Br}(C)).\right)$ 
Lemme 7.2. Soit $X / C$ une surface fibrée en coniques. Supposons qu'il existe un point $k$-rationnel de $C$ à fibre lisse. Soit $\hat{T}=\mathrm{NS}(\bar{X})$. Le diagramme

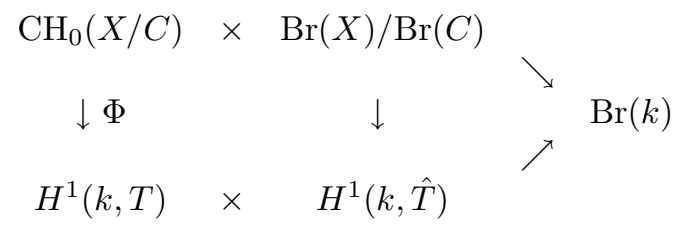

est commutatif. Dans ce diagramme, où les groupes dans le carré sont annulés par 2, l'accouplement supérieur est induit par l'évaluation $\mathrm{CH}_{0}(X) \times \mathrm{Br}(X) \rightarrow \operatorname{Br}(k)$, et l'accouplement inférieur par le cup-produit.

Démonstration. Soit $A_{0}(X) \subset \mathrm{CH}_{0}(X)$ le sous-groupe des classes de zéro-cycles de degré zéro. L'application $\Phi$ est obtenue par restriction de l'application

$$
\Phi: A_{0}(X) \rightarrow \operatorname{Ext}_{\mathcal{G}}^{1}\left(\operatorname{Pic}(\bar{X}), \bar{k}^{*}\right)
$$

définie dans [CT/S81] (voir aussi [Fro98], §1). L'application $\operatorname{Br}(X) \rightarrow H^{1}(k, \hat{T})$ dans le diagramme ci-dessus est induite par l'application

$$
\theta: \operatorname{Br}_{1}(X) \rightarrow H^{1}(\mathcal{G}, \operatorname{Pic}(\bar{X}))
$$

déduite de la suite spectrale mentionnée ci-dessus (on a ici $\operatorname{Br}_{1}(X)=\operatorname{Br}(X)$ ). Il suffit donc d'établir la commutativité (au signe près) du diagramme

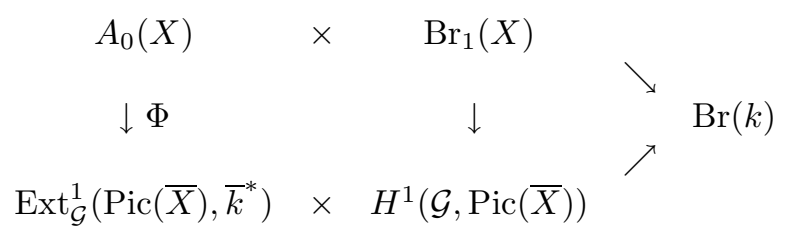

où l'accouplement $\mathrm{du}$ bas est l'accouplement évident: on décrit un élément du groupe de gauche comme une extension de (modules galoisiens) de $\operatorname{Pic}(\bar{X}) \operatorname{par} \bar{k}^{*}$; de cette extension on tire un bord allant de $H^{1}(\mathcal{G}, \operatorname{Pic}(\bar{X}))$ dans $H^{2}\left(\mathcal{G}, \bar{k}^{*}\right)=\operatorname{Br}(k)$. Le groupe $\operatorname{Br}_{1}(X)$ s'identifie au noyau de l'application $H^{2}\left(\mathcal{G}, \bar{k}(X)^{*}\right) \rightarrow H^{2}(\mathcal{G}, \operatorname{Div}(\bar{X}))$ (cf. [CT/S77], Lemme 14, p.213, ou [CT/S87], p.386). Par ailleurs on a la suite de modules galoisiens:

$$
0 \rightarrow \bar{k}(X)^{*} / \bar{k}^{*} \rightarrow \operatorname{Div}(\bar{X}) \rightarrow \operatorname{Pic}(\bar{X}) \rightarrow 0
$$

qui donne naissance à un isomorphisme

$$
H^{1}(\mathcal{G}, \operatorname{Pic}(\bar{X})) \simeq \operatorname{Ker}\left[H^{2}\left(\mathcal{G}, \bar{k}(X)^{*} / \bar{k}^{*}\right) \rightarrow H^{2}(\mathcal{G}, \operatorname{Div}(\bar{X}))\right]
$$

On montre que l'isomorphisme inverse est compatible (au signe près) avec l'application $\theta$, et ceci établit la commutativité (au signe près) du diagramme ci-dessus (pour plus de détails, voir [CT/S77], $§ 7$ et Annexe, [CT/S81], §1, et [CT/S87], Prop. 2.7.10, p.448).

Nous pouvons maintenant expliquer le lien entre les théorèmes 1.3 et 1.4. Supposons $k$ totalement imaginaire.

Pour toute place $v$ de $k$, l'accouplement de cup-produit

$$
H^{1}\left(k_{v}, T\right) \times H^{1}\left(k_{v}, \hat{T}\right) \rightarrow \operatorname{Br}\left(k_{v}\right) \hookrightarrow \mathbf{Q} / \mathbf{Z}
$$


induit un isomorphisme $H^{1}\left(k_{v}, T\right) \stackrel{\simeq}{\rightarrow} \operatorname{Hom}\left(H^{1}\left(k_{v}, \hat{T}\right), \mathbf{Q} / \mathbf{Z}\right.$ ) (théorie du corps de classes local, Tate-Nakayama). Les lemmes 7.1 et 7.2 fournissent alors pour toute place $v$ de $k$ le diagramme commutatif

$$
\begin{array}{cccc}
\mathrm{CH}_{0}\left(X_{v} / C_{v}\right) & \longrightarrow & \operatorname{Hom}\left(\operatorname{Br}\left(X_{v}\right) / \operatorname{Br}\left(C_{v}\right), \mathbf{Q} / \mathbf{Z}\right) \\
\downarrow \Phi_{v} & & \uparrow & \simeq \\
H^{1}\left(k_{v}, T\right) & \stackrel{\simeq}{ } & \operatorname{Hom}\left(H^{1}\left(k_{v}, \hat{T}\right), \mathbf{Q} / \mathbf{Z}\right) .
\end{array}
$$

Ces mêmes lemmes montrent que ce diagramme s'insère dans le diagramme commutatif:

$$
\begin{array}{ccccc}
\mathrm{CH}_{0}\left(X_{v} / C_{v}\right) & \rightarrow & \operatorname{Hom}\left(\operatorname{Br}\left(X_{v}\right) / \operatorname{Br}\left(C_{v}\right), \mathbf{Q} / \mathbf{Z}\right) & \rightarrow & \operatorname{Hom}(\operatorname{Br}(X) / \operatorname{Br}(C), \mathbf{Q} / \mathbf{Z}) \\
\downarrow & & \uparrow & \simeq & \uparrow \\
H^{1}\left(k_{v}, T\right) & \stackrel{\simeq}{\rightarrow} & \operatorname{Hom}\left(H^{1}\left(k_{v}, \hat{T}\right), \mathbf{Q} / \mathbf{Z}\right) & \rightarrow & \operatorname{Hom}\left(H^{1}(k, \hat{T}), \mathbf{Q} / \mathbf{Z}\right)
\end{array}
$$

où les flèches horizontales de droite sont déduites des flèches de restriction de $k$ à $k_{v}$.

Soit $K / k$ une extension finie, de groupe $G$, déployant le $k$-tore $T$. Soit $\mathbb{A}_{K}$ l'anneau des adèles de $K$. La flèche diagonale $T(K) \rightarrow T\left(\mathbb{A}_{K}\right)$ définit une suite exacte de modules galoisiens

$$
0 \rightarrow T(K) \rightarrow T\left(\mathbb{A}_{K}\right) \rightarrow C_{K}(T) \rightarrow 0 .
$$

Les théories du corps de classes locale et globale (Tate, Nakayama) identifient la cohomologie galoisienne des modules $T\left(\mathbb{A}_{K}\right)$ et $C_{K}(T)$. En particulier, de la suite ci-dessus on tire la suite exacte

$$
H^{1}(k, T) \rightarrow \bigoplus_{v \in \Omega} H^{1}\left(k_{v}, T\right) \rightarrow \operatorname{Hom}\left(H^{1}(k, \hat{T}), \mathbf{Q} / \mathbf{Z}\right) \rightarrow H^{2}(k, T) \rightarrow \bigoplus_{v \in \Omega} H^{2}\left(k_{v}, T\right) .
$$

Pour $v \in \Omega$, la flèche $H^{1}\left(k_{v}, T\right) \rightarrow \operatorname{Hom}\left(H^{1}(k, \hat{T}), \mathbf{Q} / \mathbf{Z}\right)$ est obtenue par composition de l'isomorphisme de dualité locale $H^{1}\left(k_{v}, T\right) \simeq \operatorname{Hom}\left(H^{1}\left(k_{v}, \hat{T}\right), \mathbf{Q} / \mathbf{Z}\right)$ et de la flèche

$$
\operatorname{Hom}\left(H^{1}\left(k_{v}, \hat{T}\right), \mathbf{Q} / \mathbf{Z}\right) \rightarrow \operatorname{Hom}\left(H^{1}(k, \hat{T}), \mathbf{Q} / \mathbf{Z}\right)
$$

duale de la restriction de $k$ à $k_{v}$.

En comparant ces divers diagrammes et suites exactes, et en tenant compte du théorème 2.4 , on voit que le théorème 1.3 implique le théorème 1.4, lorsque la courbe $C$ possède un point $k$-rationnel à fibre lisse.

Pour établir le cas général, on utilise un argument de trace. Soit $M \in C(k)$. Le zéro-cycle $M$ est rationnellement équivalent, sur $C$, à un zéro-cycle $\sum_{j \in J} n_{j} M_{j}$ dont le support est étranger aux points $P_{i}$. Le degré du zéro-cycle $\sum_{j \in J} n_{j} M_{j}$ étant égal à 1 , l'un des $M_{j}$, soit $M_{j_{0}}$, a un degré $\left[k\left(M_{j_{0}}\right): k\right]$ impair. Soit $K=k\left(M_{j_{0}}\right)$. La restriction de $k$ à $K$ envoie le complexe de groupes apparaissant dans l'énoncé du théorème 1.4 dans le complexe analogue pour $X_{K} / C_{K}$, et la corestriction envoie le complexe pour $X_{K} / C_{K}$ dans celui pour $X / C$. Comme les groupes impliqués sont tous annulés par 2, le composé de la corestriction avec la restriction est l'identité. On sait déjà que le complexe pour $X_{K} / C_{K}$ est exact. Il en est donc de même du complexe pour $X / C$.

Notons $\mathrm{CH}_{0}\left(X_{v} / C_{v}\right) / \mathrm{Br}$ le quotient de $\mathrm{CH}_{0}\left(X_{v} / C_{v}\right)$ par la relation d'équivalence induite par l'accouplement avec le groupe $\operatorname{Br}\left(X_{v}\right)$ (i.e. avec $\operatorname{Br}\left(X_{v}\right) / \operatorname{Br}\left(C_{v}\right)$ ). Le théorème 1.4 implique le théorème suivant, qu'on comparera à [CT/SwD94], Thm. 6.2, et [CT/Sk/SwD97], Thm. 4.8: 
Théorème 7.3. Soit $k$ un corps de nombres totalement imaginaire. Soit $X / C$ une surface fibrée en coniques sur $k$. Supposons $C(k) \neq \emptyset$. Alors la suite naturelle de groupes abéliens finis

$$
\mathrm{CH}_{0}(X / C) \rightarrow \bigoplus_{v \in \Omega} \mathrm{CH}_{0}\left(X_{v} / C_{v}\right) / \mathrm{Br} \rightarrow \operatorname{Hom}(\operatorname{Br}(X) / \operatorname{Br}(C), \mathbf{Q} / \mathbf{Z})
$$

est exacte.

Remarques. (a) Je ne sais pas si les théorèmes 1.4 et 7.3 valent pour un corps de nombres $k$ formellement réel.

(b) Lorsque $C$ n'est pas de genre zéro, l'application

$$
\Phi_{v}: \mathrm{CH}_{0}\left(X_{v} / C_{v}\right) \rightarrow H^{1}\left(k_{v}, T\right) \simeq \operatorname{Hom}\left(\operatorname{Br}\left(X_{v}\right) / \operatorname{Br}\left(C_{v}\right), \mathbf{Q} / \mathbf{Z}\right)
$$

n'est pas nécessairement injective (Parimala/Suresh $[\mathrm{Pa} / \mathrm{Su} 95]$ ). Le théorème 7.3 n'implique donc pas a priori le théorème 1.4, à la différence du cas des fibrés en coniques au-dessus de la droite projective (Salberger [Sal88], démonstration du théorème (7.5); voir aussi [CT/SwD94]).

(c) Supposons $X(k) \neq \emptyset$. Le théorème 1.4 est une version relative de la conjecture 1.2 de l'introduction pour $X / C$. Sous l'hypothèse que le groupe de TateShafarevich de la jacobienne de $C$ est fini, la conjecture 1.2 de l'introduction est connue pour $C$. Je ne vois pas comment combiner ces deux résultats, ou modifier la méthode, pour obtenir la conjecture 1.2 pour $X$.

\section{DÉMONSTRATION DU THÉORÈME 1.5}

Rappelons-en l'énoncé:

Theorem 1.5. Soient $k$ un corps de nombres totalement imaginaire, et $X / C$ une surface fibrée en coniques sur $k$. Supposons:

(i) La courbe $C$ est de genre un.

(ii) Le quotient $\operatorname{Br}(X) / p^{*} \operatorname{Br}(C)=0$.

(iii) Le groupe de Tate-Shafarevich de la jacobienne de $C$ est fini.

$S$ 'il existe une famille $\left\{z_{v}\right\}_{v \in \Omega}$ de zéro-cycles de degré un orthogonale au groupe de Brauer de $X$ par l'accouplement (1.1):

$$
\forall \alpha \in \operatorname{Br}(X), \quad \sum_{v \in \Omega} \operatorname{inv}_{v}\left(\alpha, z_{v}\right)=0 \in \mathbf{Q} / \mathbf{Z},
$$

alors il existe un zéro-cycle de degré un sur $X$.

Démonstration. Par $\Omega$ on désignera dans cette démonstration l'ensemble des places non archimédiennes de $k$ (ceci n'affecte pas l'hypothèse). La famille de zéro-cyles de degré un $\left\{p_{*}\left(z_{v}\right)\right\}_{v \in \Omega}$ sur $C$ annule tout élément de $\operatorname{Br}(C)$. Fixons un entier $n>0$. L'hypothèse que le groupe de Tate-Shafarevich de la jacobienne de $C$ est fini et des arguments dus à Cassels et Manin ("suite duale" de Cassels-Tate, voir ([CT99], Prop. 3.3) impliquent alors d'une part l'existence d'un point rationnel sur la courbe de genre un $C$, qu'on peut donc identifier à sa jacobienne, puis l'existence d'un point $k$-rationnel $P$ sur $C$ et pour chaque $v \in \Omega$ d'un zéro-cycle $t_{v}$ de degré zéro sur $C_{v}$ tels que l'on ait, sur chaque $C_{v}$, l'équivalence rationnelle

$$
P-p_{*}\left(z_{v}\right) \sim 4 t_{v} .
$$


Les points $P$ et les cycles $t_{v}$ dépendent de $n$, et ne sont pas uniquement déterminés. Pour la suite de l'argument, nous fixons $n=4$, puis un $k$-point $P$ associé, que nous notons $P_{\infty}$. On a donc, pour chaque place $v \in \Omega$,

$$
P_{\infty}-p_{*}\left(z_{v}\right) \sim 4 t_{v} .
$$

Si la conique $X_{\infty}=p^{-1}\left(P_{\infty}\right)$ possède un $k$-point, il en est de même de $X$ et le théorème est démontré. On supposera dans la suite $X_{\infty}(k)=\emptyset$, en particulier $X_{\infty}$ lisse.

Pour toute fibration en conique $X / C$ et tout point fermé $M$ de $C$, la fibre $X_{M}=$ $p^{-1}(M)$ est une conique sur le corps $k(M)$, elle contient donc un point fermé de degré 2 sur $k(M)$. Ainsi le zéro-cycle $2 M$ est de la forme $p_{*}(z)$ pour $z$ un zéro-cycle sur $p^{-1}(M) \subset X$. Pour toute place $v \in \Omega$, il existe donc un zéro-cycle de degré zéro $u_{v}$ sur $X_{U_{v}}$ tel qu'on ait, sur $C_{v}$, l'équivalence rationnelle

$$
P_{\infty} \sim p_{*}\left(z_{v}+2 u_{v}\right) \text {. }
$$

D'après la proposition 3.3 (Frossard/Suresh), il existe un entier $N>0$ tel que, pour tout corps $L$ contenant $k$, tout zéro-cycle $z$ sur $X_{L}$ de degré au moins égal à $N$ est rationnellement équivalent, sur $X_{L}$, à un zéro-cycle effectif $z^{\prime}$ tel que $p_{*}\left(z^{\prime}\right)$ ait toutes ses multiplicités égales à un et ait son support étranger aux points $P_{i}$ et à $P_{\infty}$.

Comme au $\S 5$, notons $g$ le genre de $C$ et $r$ le nombre de fibres géométriques singulières. Soit $m \in \mathbf{N}$ tel que $n=1+2 m \geq N$ et $n>2 g-2+r$. Soit $A$ un point de degré 2 sur $X_{\infty}$. Par la proposition 3.3, pour chaque place $v$, le cycle $z_{v}+2 u_{v}+m A$ est rationnellement équivalent, sur $X_{v}=X \times_{k} k_{v}$, à un zéro-cycle effectif $z_{v}^{\prime}$, de degré $n$, tel que $p_{*}\left(z_{v}^{\prime}\right)$ ait ses multiplicités égales à 1 et son support étranger aux points $M_{i}$ et à $P_{\infty}$. On a donc l'équivalence rationnelle

$$
n P_{\infty} \sim p_{*}\left(z_{v}^{\prime}\right)
$$

$\operatorname{sur} C_{v}$, et il existe une fonction rationnelle $f_{v} \in k_{v}[U]=H^{0}\left(U_{v}, \mathcal{O}_{U_{v}}\right)$, telle que $v_{P_{\infty}}(f)=-n$, telle que

$$
\operatorname{div}_{U_{v}}\left(f_{v}\right)=p_{*}\left(z_{v}^{\prime}\right)
$$

et telle que de plus les zéros de $f_{v}$ sur $U_{v}$ soient tous simples et étrangers aux points $M_{i}$. Par définition, $f_{v}$ appartient à $k_{v}\left(U_{v}\right)_{\mathrm{dn}}^{*}$.

Assertion. Il existe un élément $\alpha \in E^{*} / k^{*} N F^{*}$ tel que pour toute place $v \in \Omega$ la restriction de $\alpha$ à $E_{v}^{*} / k_{v}^{*} N F_{v}^{*}$ coüncide avec $\widetilde{s p}\left(f_{v}\right) \in E_{v}^{*} / k_{v}^{*} N F_{v}^{*}$.

Démonstration. De la suite (2.10) sur $k$ et sur chaque $k_{v}$, on déduit le diagramme commutatif de suites exactes

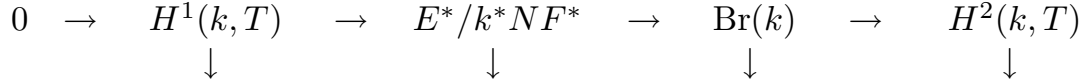

$$
\begin{aligned}
& 0 \rightarrow \bigoplus_{v \in \Omega} H^{1}\left(k_{v}, T\right) \rightarrow \bigoplus_{v \in \Omega} E_{v}^{*} / k_{v}^{*} N F_{v}^{*} \rightarrow \bigoplus_{v \in \Omega} \operatorname{Br}\left(k_{v}\right) \rightarrow \bigoplus_{v \in \Omega} H^{2}\left(k_{v}, T\right) .
\end{aligned}
$$

D'après le lemme 7.1 et l'hypothèse (ii) du théorème on a $H^{1}(k, \hat{T})=0$. La suite exacte (7.1) (Tate-Nakayama) montre alors que, dans le diagramme ci-dessus, la flèche verticale de gauche est surjective et la flèche verticale de droite injective. D'après le lemme 2.2, appliqué à chaque corps $k_{v}$, l'image de $\beta_{v}=\widetilde{s p}\left(f_{v}\right) \in$ $E_{v}^{*} / k_{v}^{*} N F_{v}^{*}$ dans $\operatorname{Br}\left(k_{v}\right)$ est égale à $-n\left[X_{\infty}\right]_{k_{v}}=\left[X_{\infty}\right]_{k_{v}}$ (rappelons que $n$ est impair). Ainsi l'image de la famille $\left\{\beta_{v}\right\}_{v \in \Omega}$ dans $\bigoplus_{v \in \Omega} \operatorname{Br}\left(k_{v}\right)$ provient-elle de la 
classe globale $\left[X_{\infty}\right] \in \operatorname{Br}(k)$. L'assertion résulte alors d'une chasse au diagramme évidente.

On peut multiplier chaque $f_{v}$ par une constante dans $k_{v}^{*}$ sans changer l'équation (8.1) ni les propriétés des fonctions $f_{v}$ (zéros simples, étrangers aux points $P_{i}$ ). L'assertion ci-dessus nous permet donc de supposer:

Il existe un élément $\eta \in E^{*} / N F^{*}$ tel que, pour toute place $v \in \Omega, s p_{v}\left(f_{v}\right) \in$ $E_{v}^{*} / N F_{v}^{*}$ coïncide avec la restriction de $\eta$.

On est alors exactement dans les conditions d'application du lemme 5.2. Avec les notations de ce lemme, il existe donc une fonction $f \in k[U]$, de degré $-v_{P_{\infty}}(f)=n$, appartenant à $k(U)_{\mathrm{dn}}^{*}$ (et telle que $s p(f)=\eta$ ). Ainsi il existe un zéro-cycle $z$ sur $U$ tel que $\operatorname{div}_{U}(f)=p_{*}(z)$. La valuation de $f$ en $P_{\infty}$ est impaire, le degré du zéro-cycle $\operatorname{div}_{U}(f)$ l'est donc aussi, celui du zéro-cycle $z$ sur $X_{U}$ l'est donc aussi. Sur $X$ il existe donc un zéro-cycle de degré impair. Comme par ailleurs $C$ contient le point rationnel $P_{\infty}$ dont la fibre est une conique lisse, il existe un point fermé de degré 2 sur $X$, si bien qu'il existe un zéro-cycle de degré 1 sur $X$.

Remarques. (a) Le quotient $\operatorname{Br}(X) / p^{*} \operatorname{Br}(C)$ est un groupe fini d'exposant 2, "souvent" nul.

(b) De manière plus générale, les hypothèses (i) et (ii), ainsi que l'hypothèse que $k$ est totalement imaginaire, sont sans doute inutiles. Notons cependant que pour $C=\mathbf{P}_{k}^{1}$, l'hypothèse $\operatorname{Br}(X) / p^{*} \operatorname{Br}(C)=0$ était faite dans [Sal88]; c'est seulement en utilisant une méthode différente qu'elle put être omise dans [CT/SwD94].

(c) A la différence du cas où $C=\mathbf{P}_{k}^{1}$, le critère donné dans le théorème n'est pas effectif, le groupe $\operatorname{Br}(C) / \operatorname{Br}(k)$ étant infini lorsque le genre de $C$ est non nul.

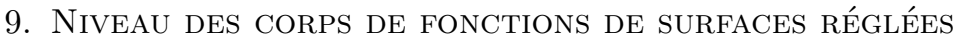

Proposition 9.1. Soient $k$ un corps de nombres, $C / k$ une $k$-courbe projective et lisse géométriquement connexe, et $X / k$ une $k$-surface projective et lisse géométriquement connexe munie d'un $k$-morphisme $p: X \rightarrow C$ de fibre générique $X_{\eta}$ une conique lisse. Soit $k(C)$ le corps des fonctions rationnelles sur $C$ et $A / k(C)$ l'algèbre de quaternions attachée à $X_{\eta}$. Supposons $X\left(k_{v}\right)=\emptyset$ pour toute place réelle $v$ de $k$. Alors -1 est une somme de quatre carrés dans $k(X)$ si et seulement s'il existe une fonction $f \in k(C)^{*}$ telle que $f \cup A=(-1,-1,-1) \in H^{3}(k(C), \mathbf{Z} / 2)$. Cette condition équivaut à l'existence de $f \in k(C)^{*}$ telle que:

a) Pour $v$ place réelle de $k, f$ est négative en tout point de $C\left(k_{v}\right)$ où elle est définie.

b) Le cup-produit $f \cup A \in H^{3}(k(C), \mathbf{Z} / 2)$ a tous ses résidus triviaux, en d'autres termes, $f$ appartient à $k(C)_{\mathrm{dn}}^{*}$, et $s p(f)=1 \in E^{*} / N F^{*}$.

c) Pour toute place finie $v$ de $k$ où la courbe $C$ a mauvaise réduction, et pour toute place 2-adique $v, f \cup A=0 \in H^{3}\left(k_{v}(C), \mathbf{Z} / 2\right)$, i.e. $f \in k_{v}(C)$ est une norme réduite de $A \otimes_{k(C)} k_{v}(C)$.

Démonstration. Par la proposition $2.3,-1$ est une somme de quatre carrés dans $k(X)$ si et seulement si $(-1,-1,-1)=0 \in H^{3}(k(X), \mathbf{Z} / 2)$. Comme $X_{\eta} / k(C)$ est une conique lisse, le noyau de l'application $H^{3}(k(C), \mathbf{Z} / 2) \rightarrow H^{3}(k(X), \mathbf{Z} / 2)$ est formé des éléments de la forme $f \cup A$ avec $f \in k(C)^{*}$ (Arason [Ar75]). Ainsi -1 est une somme de quatre carrés dans $k(X)$ si et seulement s'il existe $f \in k(C)^{*}$ tel que $(-1,-1,-1)=f \cup A \in H^{3}(k(C), \mathbf{Z} / 2)$. 
S'il existe une telle fonction $f$, tous les résidus de $f \cup A$ sont triviaux. Ceci est le cas si et seulement si $f$ appartient à $k(C)_{\mathrm{dn}}^{*}$ et de plus $s p(f)=1 \in E^{*} / N F^{*}$. Par ailleurs, pour $v$ place finie, $(-1,-1,-1)=0 \in k_{v}$, on a donc $f \cup A=0 \in$ $H^{3}\left(k_{v}(C), \mathbf{Z} / 2\right)$. En utilisant les résultats de Witt sur les courbes sur le corps des réels, on établit le:

Lemme 9.2. Soit $X \rightarrow C$ une fibration en coniques au-dessus d'une courbe, le corps de base étant le corps $\mathbf{R}$ des réels. Soit $A / \mathbf{R}(C)$ l'algèbre de quaternions associée. Si $X(\mathbf{R})=\emptyset$, alors $X / C$ est birationnel (au-dessus de $C$ ) au produit de $C$ et de la conique d'équation homogène $x^{2}+y^{2}+z^{2}=0$, et l'algèbre $A$ est $\mathbf{R}(C)$-isomorphe à l'algèbre de quaternions standard $(-1,-1)$.

Pour $v$ place réelle de $k$, et $X / C$ comme dans la proposition, on a donc

$$
A \otimes_{k(C)} k_{v}(C)=(-1,-1)_{k_{v}(C)}
$$

et donc pour $f$ comme ci-dessus, $(f,-1,-1)=(-1,-1-1) \in H^{3}\left(k_{v}(C), \mathbf{Z} / 2\right)$. Mais alors (proposition 2.3), $-f$ est une somme de quatre carrés dans $k_{v}(C)$, ce qui par les résultats de Witt équivaut aussi au fait que $-f$ est positive sur $C\left(k_{v}\right)$ là où elle est définie, ou encore au fait que $-f$ est une somme de deux carrés dans $k_{v}(C)$.

Réciproquement, soit $f$ comme dans l'énoncé du théorème. La classe $f \cup A \in$ $H^{3}(k(C), \mathbf{Z} / 2)$ est non ramifiée en tout point fermé de $C$. Ceci implique le même énoncé pour l'image de $f \cup A$ dans chaque $H^{3}\left(k_{v}(C), \mathbf{Z} / 2\right)$. Cette image est donc nulle pour toute place finie $v$, par hypothèse pour les places de mauvaise réduction, et par [Kato86], Cor. 2.9, pour les autres places. L'hypothèse a) et les résultats de Witt assurent que pour toute place réelle $v$ de $k$, la classe de $f \cup A$ coïncide avec celle de $(-1,-1,-1)$ dans $H^{3}\left(k_{v}(C), \mathbf{Z} / 2\right)$. Ainsi $f \cup A \in H^{3}(k(C), \mathbf{Z} / 2)$ et $(-1,-1,-1) \in H^{3}(k(C), \mathbf{Z} / 2)$ ont même image dans $H^{3}\left(k_{v}(C), \mathbf{Z} / 2\right)$ pour chaque place $v$ de $k$. D'après le principe local-global de Kato ([Kato86], Theorem $0.8(2)$ ), ceci assure $f \cup A=(-1,-1,-1) \in H^{3}(k(C), \mathbf{Z} / 2)$. Ainsi $(-1,-1,-1)=0 \in$ $H^{3}(k(X), \mathbf{Z} / 2)$, et, par la proposition $2.3,-1$ est une somme de quatre carrés dans le corps $k(X)$.

On sait [CT/J91] que si $X / k$ est une surface projective, lisse, géométriquement intègre sur un corps de nombres $k$, telle que $X\left(k_{v}\right)=\emptyset$ pour toute place réelle $v$ de $k$, alors -1 est une somme de 8 carrés dans le corps des fonctions de $X$. Pour les surfaces fibrées en coniques, on peut dire plus.

Théorème 9.3. Soit $X / C$ une fibration en coniques au-dessus d'une courbe sur un corps de nombres. Supposons $C(k) \neq \emptyset$, et supposons $X\left(k_{v}\right)=\emptyset$ pour toute place réelle $v$ de $k$. Alors -1 est une somme de quatre carrés dans $k(X)$.

Démonstration. On peut supposer que $k$ a au moins une place réelle, sinon l'énoncé est clair. La fibre $X_{\infty}$ est lisse (sinon elle aurait un $k$-point, et donc un $k_{v}$-point pour $v$ place réelle). Soit $A$ un point fermé de degré 2 sur la fibre $X_{\infty}$. Procédant comme au $\S 3$, on trouve une courbe $D \subset X$, lisse et géométriquement intègre, finie sur $C$, et un zéro-cycle effectif $z$ à support dans $X \backslash X_{\infty}$, tel que $z$ soit rationnellement équivalent à $s A$ sur $D$, avec $n=2 s>2 g-2+r$ (notations du lemme 5.1), et que $p_{*}(z)$ ait toutes ses multiplicités égales à 1 , et son support en dehors des points $P_{i}, i \in I$. Soit $g \in k(D)$ telle que $\operatorname{div}_{D}(g)=z-s A$. Soit $h$ la norme de $k(D)$ à $k(C)$ de $g$. C'est une norme réduite de $A$, et $\operatorname{div}_{D}(h)=p_{*} z-2 s P_{\infty}$. 
Pour chaque place $v$ finie ou complexe de $k$, définissons $f_{v}=h \in k_{v}(C)$. Pour $v$ place réelle, soit $f_{v}=-h$. Les fonctions $f_{v}$ satisfont les hypothèses du lemme 5.2 , avec $\eta=1 \in E^{*} / N_{E / F} F^{*}$. Pour $v$ finie ou complexe, c'est évident. Pour $v$ réel, il est clair que $-h$ appartient à $k_{v}(C)_{\mathrm{dn}}^{*}$. De l'hypothèse $X\left(k_{v}\right)=\emptyset$ il résulte $E_{v}^{*} / N F_{v}^{*}=$ 1. On a donc bien aussi $s p\left(f_{v}\right)=1$. Soit $S_{0}$ un ensemble fini de places contenant les places réelles, les places dyadiques et les places finies de mauvaise réduction pour $C$. Le lemme principal 5.2 et sa démonstration assurent alors l'existence d'une fonction $f \in V_{n} \subset k[U]$ appartenant à $k(U)_{\mathrm{dn}}^{*}$, telle que $s p(f)=1$, donc telle que $f \cup A \in H^{3}(k(C), \mathbf{Z} / 2)$ soit non ramifié en tout point de $U$, donc de $C$ par réciprocité, telle que de plus $f / f_{v} \in \operatorname{Nrd}\left(A_{k_{v}(C)}\right)$ pour chaque place $v$, et qu'enfin $f \in V_{n} \otimes_{k} k_{v}$ soit arbitrairement proche de $f_{v}=-h$ pour $v$ réelle. Soit $v$ une place réelle. De l'hypothèse $X\left(k_{v}\right)=\emptyset$ et du fait que $p_{*}(z)$ a toutes ses multiplicités égales à 1 , on conclut que $h$ n'a pas de zéro ou pôle dans $C\left(k_{v}\right)$ autre que le point $P_{\infty}$, en outre $h$ est une norme réduite de $A$, c'est donc une somme de quatre carrés dans $k_{v}(C)$ (lemme 9.2), et donc strictement positive sur $U\left(k_{v}\right)$. Par une variante du lemme de Krasner, on voit que si $f \in V_{n} \otimes_{k} k_{v}$ est assez proche de $f_{v}=-h$, alors $f$ est strictement négative en tout point de $U\left(k_{v}\right)$. Les conditions de la proposition 9.1 sont donc satisfaites, et -1 est une somme de quatre carrés dans $k(C)$.

\section{REFERENCES}

[Ar75] J. Kr. Arason, Kohomologische Invarianten quadratischer Formen, J. Algebra 36 (1975), 448-491. MR 52:10592

[Ca64] J.W.S. CAssels, Arithmetic on curves of genus 1 (VII). The dual exact sequence, J. für die reine und angew. Math. (Crelle) 216 (1964), 150-158. MR 30:92

[CT95] J.-L. Colliot-ThÉLÈne, L'arithmétique du groupe de Chow des zéro-cycles, Journées arithmétiques de Bordeaux 1993, Journal de théorie des nombres de Bordeaux 7 (1995), 51-73. MR 97i:14006

[CT99] J.-L. Colliot-ThÉLÈne, Conjectures de type local-global sur l'image de l'application cycle en cohomologie étale, to appear in Proceedings of the 1997 Seattle conference on Algebraic K-Theory, W. Raskind and C. Weibel, eds., Symposia in Pure Mathematics 67, Amer. Math. Soc., Providence, 1999.

[CT/J91] J.-L. Colliot-ThÉlène et U. JAnnsen, Sommes de carrés dans les corps de fonctions, C. R. Acad. Sci. Paris 312 (1991), 759-762. MR 92c:11036

[CT/S77] J.-L. Colliot-ThÉlène et J.-J. Sansuc, La R-équivalence sur les tores, Ann. Scient. Éc. Norm. Sup. 10 (1977), 175-229. MR 56:8576

[CT/S81] J.-L. Colliot-ThÉLÈnE et J.-J. SANSUC, On the Chow groups of certain rational surfaces: a sequel to a paper of S. Bloch, Duke Math. J. 48 (1981), 421-447. MR 83e: 14007

[CT/S87] J.-L. Colliot-ThÉLÈne et J.-J. SAnsuc, La descente sur les variétés rationnelles II, Duke Math. J. 54 (1987), 375-492. MR 89f: 11082

[CT/Sko93] J.-L. Colliot-Thélène et A.N. Skorobogatov, Groupe de Chow des zérocycles sur les fibrés en quadriques, K-Theory 7 (1993), 477-500. MR 95c:14012

[CT/Sk/SwD97] J.-L. Colliot-Thélène, A.N. Skorobogatov et Sir Peter Swinnerton-Dyer, Rational points and zero-cycles on fibred varieties: Schinzel's hypothesis and Salberger's device, J. für die reine und angew. Math. (Crelle) 495 (1998), 1-28. MR 99i: 14027

[CT/SwD94] J.-L. Colliot-Thélène et Sir Peter Swinnerton-Dyer, Hasse principle and weak approximation for pencils of Severi-Brauer varieties and similar varieties, J. für die reine und angew. Math. (Crelle) 453 (1994), 49-112. MR 95h:11060

[Co80] D. F. CoRAY, Two remarks on the Bertini theorem, tapuscrit 1980.

[Fro98] E. Frossard, Groupe de Chow de dimension zéro des fibrations en variétés de Severi-Brauer, Compositio Math. 110 (1998), 187-213. MR 99c:14011 
[Fr/Su99] E. Frossard et V. Suresh, Un lemme de déplacement pour les zéro-cycles sur les fibrations en coniques, appendice au présent article, J. Amer. Math. Soc., ce volume.

[Gros87] M. Gros, 0-cycles de degré 0 sur les surfaces fibrées en coniques, J. für die reine und angew. Math. (Crelle) 373 (1987), 166-184. MR 88h:14007

[Kato86] K. KAто, A Hasse principle for two-dimensional global fields, J. für die reine und angew. Math. (Crelle) 366 (1986), 142-181. MR 88b:11036

[Kl/Al79] S. L. Kleiman et A. B. Altman, Bertini theorems for hypersurface sections containing a subscheme, Communications in Algebra 7 (8) (1979), 775-790. MR 81i: 14007

[Ma70] Yu. I. MANin, Le groupe de Brauer-Grothendieck en géométrie diophantienne, in Actes du Congrès International des Mathématiciens, Nice, 1970, Tome I, 401-411. MR 55:356

[M/S82] A. S. Merkur'Ev et A. A. Suslin, K-cohomologie des variétés de Severi-Brauer et homomorphisme de norme résiduel (en russe), Izv. Akad. Nauk SSSR, Ser. Mat. 46 (1982); trad. ang. Math. USSR Izvestija 21 (1983), 307-340. MR 84i: 12007

[Milne86] J. S. Milne, Arithmetic Duality Theorems, Perspectives in Math., vol. 1, Academic Press, 1986. MR 88e:14028

[Pa/Su95] R. PARImala et V. Suresh, Zero-cycles on quadric fibrations: Finiteness theorems and the cycle map, Invent. math. 122 (1995), 83-117. MR 96m:14009

[Sai89] S. SAITo, Some observations on motivic cohomology of arithmetic schemes, Invent. math. 98 (1989), 371-404. MR 90k:11077

[Sal85] P. SAlbergen, K-theory of orders and their Brauer-Severi schemes, Thèse, Université de Göteborg, 1985.

[Sal88] P. SAlberger, Zero-cycles on rational surfaces over number fields, Invent. math. 91 (1988), 505-524. MR 89c: 14010

[San82] J.-J. SANSuc, Descente et principe de Hasse pour certaines variétés rationnelles, Séminaire de théorie des nombres, Paris 1980-1981, Progress in Math., vol. 22, Birkhäuser, 1982. MR 84h: 14026

[Se64] J.-P. SerRe, Lie Algebras and Lie Groups, Benjamin 1965. 2nd ed., Lecture Notes in Mathematics, vol. 1500, Springer-Verlag, 1992. MR 93h:17001

[Witt37] E. WiтT, Zerlegung reeller algebraischer Funktionen in Quadrate. Schiefkörper über reellem Funktionenkörper, J. für die reine und angew. Math. (Crelle) 171 (1934), 31-44.

C.N.R.S., UMR 8628, Mathématiques, BÂtiment 425, Université de Paris-Sud, F-91405 ORSAY, FRANCE

E-mail address: colliot@math.u-psud.fr 